\title{
Divergent Reactivity via Cobalt Catalysis: An \\ Epoxide Olefination
}

\author{
Megan L. Jamieson, Paul A. Hume, Daniel. P. Furkert* and Margaret A. Brimble* \\ School of Chemical Sciences, University of Auckland, 23 Symonds Street, Auckland 1142, \\ New Zealand; Maurice Wilkins Centre for Molecular Biodiscovery
}

\begin{abstract}
Contents
$\begin{array}{ll}\text { Experimental procedures } & \mathrm{S} 2\end{array}$

$\begin{array}{ll}{ }^{1} \mathrm{H} \text { and }{ }^{13} \mathrm{C} \text { NMR spectra } & \mathrm{S} 18\end{array}$

${ }^{19}$ F NMR spectra of Mosher esters $\quad$ S34

$\begin{array}{ll}\text { HPLC traces } & \text { S35 }\end{array}$
\end{abstract}




\section{General Experimental}

Unless otherwise noted, all reactions were performed under an oxygen-free atmosphere of nitrogen using standard techniques. All reagents were used as received unless otherwise noted. Yields refer to chromatographically and spectroscopically ( ${ }^{1} \mathrm{H}$ NMR) homogeneous materials, unless otherwise stated. Reactions were monitored by thinlayer chromatography (TLC) carried out on silica gel plates using UV light as visualizing agent and an ethanolic solution of vanillin and ammonium molybdate and heat as developing agents. Silica gel $(60,230-400$ mesh) was used for flash column chromatography. Melting points are uncorrected. Optical rotations were measured with an Autopol® IV automatic polarimeter, using the Na-D line $(589 \mathrm{~nm})$ with the concentration of the solution measured in g/100 mL. Infrared (IR) spectra were recorded using a Perkin Elmer Spectrum 100 FT-IR spectrometer on a film ATR sampling accessory. Absorption maxima are expressed in wavenumbers $\left(\mathrm{cm}^{-1}\right)$ and recorded using a range of $450-4000 \mathrm{~cm}^{-1}$. NMR spectra were recorded at room temperature in $\mathrm{CDCl}_{3}$, on a spectrometer operating at $400 \mathrm{MHz}$ for ${ }^{1} \mathrm{H}$ nuclei, $100 \mathrm{MHz}$ for ${ }^{13} \mathrm{C}$ nuclei, and $376 \mathrm{MHz}$ for ${ }^{19} \mathrm{~F}$ nuclei. Chemical shifts are reported in parts per million (ppm) on the $\delta$ scale and coupling constants, $J$, are in hertz $(\mathrm{Hz})$. Multiplicities are reported as "s" (singlet), "br s" (broad singlet), “d” (doublet), "dd" (doublet of doublets), "t" (triplet), "m" (multiplet), "ABq" (AB quartet), and $\mathrm{ABX} .{ }^{1} \mathrm{H}$ and ${ }^{13} \mathrm{C}$ NMR resonances were assigned using a combination of DEPT 135, COSY, HSQC, HMBC, and NOESY spectra. High-resolution mass spectra (HRMS) were obtained using a Bruker micrOTOF-Q II mass spectrometer operating at a nominal accelerating voltage of $70 \mathrm{eV}$. 




$( \pm)-7$

\section{2-Benzyloxymethyloxirane $(( \pm)-7)$}

To ( \pm )-epichlorohydrin $(4.2 \mathrm{~mL}, 54.0 \mathrm{mmol})$ and tetrabutylammoniumbromide $(0.355 \mathrm{~g}$, $1.10 \mathrm{mmol})$ in aqueous $\mathrm{NaOH}(9.3 \mathrm{~mL}, 50 \% \mathrm{w} / \mathrm{w})$ was added benzyl alcohol $(5.6 \mathrm{~mL}$, $54.0 \mathrm{mmol}$ ) dropwise over $30 \mathrm{~min}$. The mixture was stirred for $20 \mathrm{~h}$, then poured into cold water $(70 \mathrm{~mL})$. The aqueous layer was extracted with EtOAc $(3 \times 50 \mathrm{~mL})$. The combined organic extracts were dried over anhydrous $\mathrm{Na}_{2} \mathrm{SO}_{4}$, filtered and concentrated in vacuo. Purification by column chromatography (hexanes/EtOAc 9:1) afforded the title compound $( \pm)-7$ (6.36 g, $38.7 \mathrm{mmol}, 72 \%)$ as a colourless oil). ${ }^{1} \mathrm{H}$ NMR (400 MHz; $\left.\mathrm{CDCl}_{3}\right) \delta=7.34-7.27(\mathrm{~m}, 5 \mathrm{H}), 4.58(\mathrm{ABq}, 2 \mathrm{H}), 3.76(\mathrm{dd}, J=11.4,3.0 \mathrm{~Hz}, 1 \mathrm{H}), 3.45$ $(\mathrm{dd}, J=11.4,6.0 \mathrm{~Hz}, 1 \mathrm{H}), 3.21-3017(\mathrm{~m}, 1 \mathrm{H}), 2.80(\mathrm{dd}, J=4.2,4.2 \mathrm{~Hz} 1 \mathrm{H}), 2.62(\mathrm{dd}$, $J=4.8,2.8 \mathrm{~Hz}, 1 \mathrm{H})$.

Spectral data in agreement with that reported in the literature. ${ }^{1}$

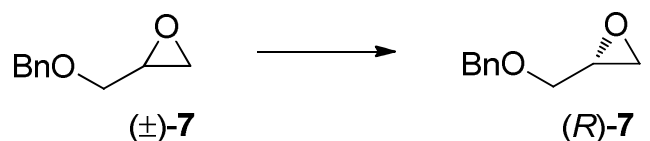

\section{$(R)$-2-Benzyloxymethyloxirane $((R)-7)$}

To $\quad(S, S)-(+)-N, N^{\prime}$-Bis(3,5-di-tert-butylsalicylidene)-1,2-cyclohexanediaminocobalt(II) $(69.9 \mathrm{mg}, 0.12 \mathrm{mmol})$ in toluene $(0.58 \mathrm{~mL})$ was added acetic acid $(20 \mu \mathrm{L}, 0.35 \mathrm{mmol})$ dropwise. The resultant mixture was stirred open to the air for $1 \mathrm{~h}$, then concentrated in vacuo. 2-Benzyloxymethyloxirane $( \pm)-7(8.67 \mathrm{~g}, 57.9 \mathrm{mmol})$ was added and the mixture cooled to $0{ }^{\circ} \mathrm{C}$. Water $(0.57 \mathrm{~mL}, 31.8 \mathrm{mmol})$ was added slowly and the mixture stirred for $48 \mathrm{~h}$ at $\mathrm{rt}$. Purification of the reaction mixture by Kugelrohr distillation afforded the title compound (R)-7 (4.16 g, $25.3 \mathrm{mmol}, 44 \%,>99 \%$ e.e.) as a colourless oil. $[\alpha]_{\mathrm{D}}{ }^{20}+1.8\left(c 1.00\right.$ in $\left.\mathrm{CHCl}_{3}\right) \operatorname{lit}^{2}+1.9\left(c 0.90\right.$ in $\left.\mathrm{CHCl}_{3}\right)$.

HPLC: column, Chiralpak IC; mobile phase, $n$-hexane/isopropanol (19: $1 \mathrm{v} / \mathrm{v})$; flow rate, $0.5 \mathrm{~mL} / \mathrm{min}$; retention times, $20.6 \mathrm{~min}(R), 23.9 \mathrm{~min}(S)$. 




\section{$(R)$-2-Benzyloxymethyloxetane $((R)-8)$}

$t$-BuOK $(2.05 \mathrm{~g}, 18.3 \mathrm{mmol})$ was dissolved in $t$-BuOH $(24 \mathrm{~mL})$. Trimethylsulfoxonium iodide $(4.02 \mathrm{~g}, 18.3 \mathrm{mmol})$ was added and the mixture heated to $60{ }^{\circ} \mathrm{C}$. $(R)$-2-Benzyloxymethyloxirane $(R)-7(1.18 \mathrm{~g}, 7.19 \mathrm{mmol})$ in $t$-BuOH $(3 \mathrm{~mL})$ was added and the resulting mixture stirred at $60{ }^{\circ} \mathrm{C}$ for $4 \mathrm{~h}$. The reaction mixture was cooled to $\mathrm{rt}$ then quenched with $\mathrm{H}_{2} \mathrm{O}(30 \mathrm{~mL})$. The aqueous layer was extracted with EtOAc $(3 \times 20 \mathrm{~mL})$. The combined organic extracts were washed with water $(100 \mathrm{~mL})$ and brine $(100 \mathrm{~mL})$ then dried over anhydrous $\mathrm{Na}_{2} \mathrm{SO}_{4}$, filtered and concentrated in vacuo. Purification by flash chromatography (hexanes/EtOAc 4:1) afforded the title compound $(R)-8(0.973 \mathrm{~g}, 5.46 \mathrm{mmol}, 76 \%)$ as a colourless oil. $[\alpha]_{\mathrm{D}}{ }^{20}+4.4\left(c 0.95\right.$ in $\left.\mathrm{CHCl}_{3}\right)$ Lit. +4.7 (c 0.009 in $\left.\mathrm{CHCl}_{3}\right) ;{ }^{1} \mathrm{H}$ NMR (400 $\left.\mathrm{MHz} ; \mathrm{CDCl}_{3}\right) \delta 7.36-7.28(5 \mathrm{H}, \mathrm{m})$, 4.99-4.96 (1H, m), 4.70-4.55 (4H, m), 3.69-3.60 (2H, m), 2.67-2.55 (2H, m).

The spectroscopic data were in agreement with that reported in the literature. ${ }^{3}$ 


\section{Representative Procedure for the Homologation of Epoxides}

A stirred suspension of epoxide (1 equiv), $(S, S)-N, N^{\prime}$-bis(3,5-di-tert-butylsalicylidene)1,2-cyclohexanediaminocobalt(II) (0.05 equiv), trimethylsulfoxonium iodide (3-4 equiv) and $t$-BuOK (3-4 equiv) in $t$ - $\mathrm{BuOH}(10 \mathrm{~mL} / \mathrm{mmol}$ epoxide) was heated at $60{ }^{\circ} \mathrm{C}$ for $24 \mathrm{~h}$. The reaction mixture was allowed to cool to room temperature, quenched by the addition of sat. aq. $\mathrm{NaHCO}_{3}$ and extracted with $\mathrm{Et}_{2} \mathrm{O}$. The combined organic extracts were dried over anhydrous $\mathrm{MgSO}_{4}$ and concentrated in vacuo.

*Known epoxides were prepared by literature methods. Preparation of novel epoxides is included. 


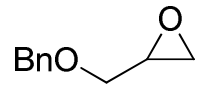

$( \pm)-7$

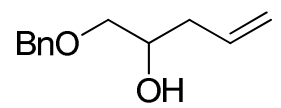

$( \pm)-9$

\section{( \pm -1-(Benzyloxy)pent-4-en-2-ol (( \pm$)-9)$}

Epoxide $7(50 \mathrm{mg}, 0.30 \mathrm{mmol})$ was subjected to the general homologation procedure. Purification by column chromatography (hexanes/EtOAc 5:1) afforded the title compound $( \pm)-9(59 \mathrm{mg}, 0.30 \mathrm{mmol}, 100 \%)$ as a colourless oil. ${ }^{1} \mathrm{H}$ NMR $(400 \mathrm{MHz}$, $\left.\mathrm{CDCl}_{3}\right): \delta 7.30-7.27(5 \mathrm{H}, \mathrm{m}, \mathrm{Ar}-\mathrm{H}), 5.87-5.76(1 \mathrm{H}, \mathrm{m}), 5.13-5.06(2 \mathrm{H}, \mathrm{m}), 4.54(2 \mathrm{H}, \mathrm{s})$, 3.89-3.84 (1H, m), $3.45(2 \mathrm{H}, \mathrm{ABX}, J=9.5,7.4,3.5 \mathrm{~Hz}), 2.50(1 \mathrm{H}, \mathrm{d}, J=3.7 \mathrm{~Hz})$, 2.27-2.23 (2H, m).

The spectroscopic data were in agreement with that reported in the literature. ${ }^{4}$

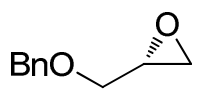

$(R)-7$

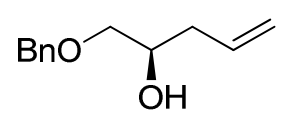

$(R)-9$

\section{(R)-1-(Benzyloxy)pent-4-en-2-ol (9)}

Epoxide $(R)-7(250 \mathrm{mg}, 1.52 \mathrm{mmol})$ was subjected to the general homologation procedure. Purification by column chromatography (hexanes/EtOAc 4:1) afforded the title compound $9(263 \mathrm{mg}, 1.37 \mathrm{mmol}, 90 \%)$ as a colourless oil. $[\alpha]_{\mathrm{D}}^{25}=-3.8(c 1.37$ in $\left.\mathrm{CHCl}_{3}\right)$ Lit. $[\alpha]_{\mathrm{D}}^{25}=-3.75\left(c 0.87\right.$ in $\left.\mathrm{CHCl}_{3}\right)$.

The spectroscopic data were in agreement with that reported in the literature. ${ }^{5}$ 

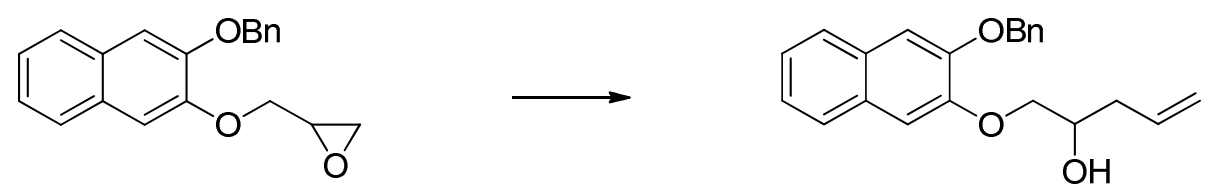

S1

\section{( \pm -1-((3-(Benzyloxy)naphthalen-2-yl)oxy)pent-4-en-2-ol (S1)}

Epoxide $^{6}(50 \mathrm{mg}, 0.16 \mathrm{mmol})$ was subjected to the general homologation procedure. Purification by column chromatography (hexanes/EtOAc 5:1) afforded the title compound S1 (45 mg, $0.13 \mathrm{mmol}, 82 \%$ ) as a pale brown solid. m.p. 69.0-71.5 ${ }^{\circ} \mathrm{C}$; ${ }^{1} \mathrm{H}$ NMR (400 MHz, $\left.\mathrm{CDCl}_{3}\right): \delta$ 7.65-7.62 $(2 \mathrm{H}, \mathrm{m}), 7.48-7.46(2 \mathrm{H}, \mathrm{m}), 7.40-7.30(5 \mathrm{H}$, m), $7.16(2 \mathrm{H}, \mathrm{d}, J=17.6 \mathrm{~Hz}), 5.92-5.84(1 \mathrm{H}, \mathrm{m}), 5.18(2 \mathrm{H}, \mathrm{s}), 5.14-5.10(2 \mathrm{H}, \mathrm{m})$, 4.15-4.10 (2H, m), 4.01-3.98 (1H, m), $2.83\left(1 \mathrm{H}\right.$, br s), 2.42-2.39 $(2 \mathrm{H}, \mathrm{m}) ;{ }^{13} \mathrm{C} \mathrm{NMR}$ $\left(100 \mathrm{MHz} \mathrm{CDCl}_{3}\right): \delta 148.9,148.8,136.7,134.0,129.5,129.2,128.6(\mathrm{CH} \times 2), 128.0$, $127.2(\mathrm{CH} \times 2), 126.4,126.3,124.4,124.3,118.0,109.4,108.9,73.1,70.6,69.2,37.7$; IR (film) $v_{\max } 3310,2925,2867,1739,1601,1509,1485,1453,1408,1382,1252,1172$, $1014,851,743 \mathrm{~cm}^{-1}$; HRMS (ESI+) for $\mathrm{C}_{22} \mathrm{H}_{22} \mathrm{NaO}_{3}[\mathrm{M}+\mathrm{Na}]^{+}$requires 357.1461 found 357.1455 .
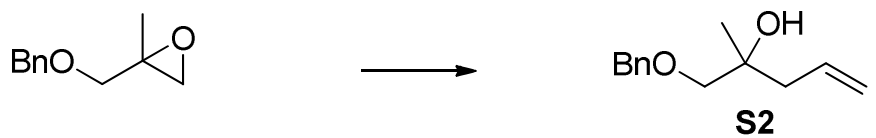

\section{( \pm -1-(Benzyloxy)-2-methylpent-4-en-2-ol (S2)}

Epoxide $^{7}$ (50 $\mathrm{mg}, 0.28 \mathrm{mmol}$ ) was subjected to the general homologation procedure. Purification by column chromatography (hexanes/EtOAc 7:1) afforded the title compound S2 (58 mg, $0.28 \mathrm{mmol}, 100 \%)$ as a colourless oil. ${ }^{1} \mathrm{H}$ NMR (400 MHz, $\left.\mathrm{CDCl}_{3}\right): \delta$ 7.29-7.19 (5H, m), 5.80-5.69 (1H, m), 5.02-4.98 (2H, m), $4.47(2 \mathrm{H}, \mathrm{s})$, $3.28-3.20(2 \mathrm{H}, \mathrm{m}), 2.32(1 \mathrm{H}$, br s$), 2.27-2.16(2 \mathrm{H}, \mathrm{m}), 1.09(3 \mathrm{H}, \mathrm{s}) ;{ }^{13} \mathrm{C} \mathrm{NMR}$ $\left(100 \mathrm{MHz}, \mathrm{CDCl}_{3}\right): \delta 138.1,133.8,128.3(\mathrm{CH} \times 2), 127.6,127.5(\mathrm{CH} \times 2), 118.2,76.8$, 73.4, 71.8, 43.6, 23.7; IR (film) $v_{\max }$ 3440, 2976, 2858, 1453, 1370, 1096, 1001, 911, $735,697 \mathrm{~cm}^{-1}$; HRMS (ESI+) for $\mathrm{C}_{13} \mathrm{H}_{18} \mathrm{NaO}_{2}[\mathrm{M}+\mathrm{Na}]^{+}$requires 229.1199 found 229.1194. 

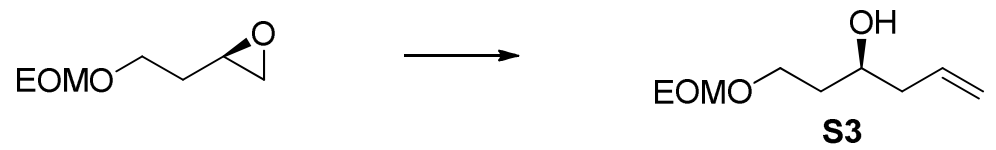

\section{(S)-1-(Ethoxymethoxy)hex-5-en-3-ol (S3)}

Epoxide $^{8}(50 \mathrm{mg}, 0.32 \mathrm{mmol})$ was subjected to the general homologation procedure. Purification by column chromatography (hexanes/EtOAc 5:1) afforded the title compound S3 (49 mg, $0.28 \mathrm{mmol}, 96 \%)$ as a yellow oil. $[\alpha]_{\mathrm{D}}^{25}=+3.1(c=0.42$ in $\left.\mathrm{CHCl}_{3}\right) ;{ }^{1} \mathrm{H} \mathrm{NMR}\left(400 \mathrm{MHz}, \mathrm{CDCl}_{3}\right): \delta 5.88-5.81(1 \mathrm{H}, \mathrm{m}), 5.15-5.10(2 \mathrm{H}, \mathrm{m}), 4.68(2 \mathrm{H}$, s), 3.86-3.85 $(1 \mathrm{H}, \mathrm{m}), 3.79-3.68(2 \mathrm{H}, \mathrm{m}), 3.60(2 \mathrm{H}, \mathrm{q}, J=7.0 \mathrm{~Hz}), 2.61(1 \mathrm{H}, \mathrm{d}$, $J=3.2 \mathrm{~Hz}), 2.29-2.24(2 \mathrm{H}, \mathrm{m}), 1.79-1.71(2 \mathrm{H}, \mathrm{m}), 1.23(3 \mathrm{H}, \mathrm{t}, J=7.2 \mathrm{~Hz}) ;{ }^{13} \mathrm{C} \mathrm{NMR}$ $\left(100 \mathrm{MHz}, \mathrm{CDCl}_{3}\right): \delta 134.7,117.7,95.1,69.8,66.0,63.4,41.9,35.9,15.1$; IR (film) $v_{\max } 3442,2976,2828,2877,1641,138,1111,1039,913,846 \mathrm{~cm}^{-1}$; HRMS (ESI+) for $\mathrm{C}_{9} \mathrm{H}_{18} \mathrm{NaO}_{3}[\mathrm{M}+\mathrm{Na}]^{+}$requires 197.1148 found 197.1155 . 


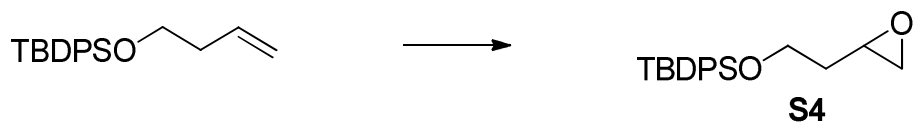

( \pm )-1-((tert-Butyldiphenylsilyl)oxy)but-3-ene oxide (S4)

To a stirred solution of 1-((tert-butyldiphenylsilyl)oxy)but-3-ene (1.88 g, $6.04 \mathrm{mmol})$ in $\mathrm{CH}_{2} \mathrm{Cl}_{2}(10 \mathrm{~mL})$ was added $m$-CPBA $(2.083 \mathrm{~g}, 7.24 \mathrm{mmol}, 60 \% \mathrm{wt} / \mathrm{wt})$ in $\mathrm{CH}_{2} \mathrm{Cl}_{2}$ $(15 \mathrm{~mL})$. The reaction mixture was stirred for $23 \mathrm{~h}$, then quenched by the addition of $1 \mathrm{M}$ aq. $\mathrm{NaOH}(25 \mathrm{~mL})$. The aqueous layer was extracted with $\mathrm{CH}_{2} \mathrm{Cl}_{2}(3 \times 30 \mathrm{~mL})$, washed with brine $(120 \mathrm{~mL})$, dried over anhydrous $\mathrm{Na}_{2} \mathrm{SO}_{4}$ and concentrated in vacuo. Purification by column chromatography (petroleum ether/EtOAc 9:1) afforded the title compound S4 (1.942 g, $5.95 \mathrm{mmol}, 99 \%)$ as a colourless oil. ${ }^{1} \mathrm{H}$ NMR (400 MHz, $\left.\mathrm{CDCl}_{3}\right): \delta 7.70-7.66(4 \mathrm{H}, \mathrm{m}), 7.43-7.36(6 \mathrm{H}, \mathrm{m}), 3.88-3.78(2 \mathrm{H}, \mathrm{m}), 3.12-3.08(1 \mathrm{H}, \mathrm{m})$, $2.78(1 \mathrm{H}, \mathrm{dd}, J=4.8,4.0 \mathrm{~Hz}), 2.53-2.51(1 \mathrm{H}, \mathrm{dd}, J=4.8,4.0 \mathrm{~Hz}), 1.80-1.76(2 \mathrm{H}, \mathrm{m})$, $1.06(9 \mathrm{H}, \mathrm{s})$.

HPLC: column, Chiralpak IC; mobile phase, $n$-hexane/isopropanol (99.95: $0.05 \mathrm{v} / \mathrm{v})$; flow rate, $0.5 \mathrm{~mL} / \mathrm{min}$; retention times, $30.2 \mathrm{~min}(S), 33.2 \mathrm{~min}(R)$.

The spectroscopic data were in agreement with that reported in the literature. ${ }^{9}$ 


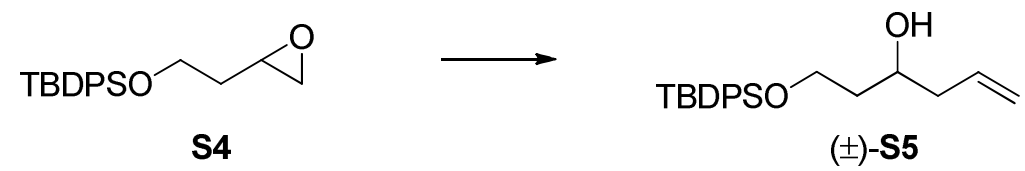

\section{( \pm )-1-((tert-Butyldiphenylsilyl)oxy)hex-5-en-3-ol (( \pm$)-S 5)$}

Epoxide $( \pm)-\mathbf{S} 4(1.306 \mathrm{~g}, 4.00 \mathrm{mmol})$ was subjected to the general homologation procedure. Purification by column chromatography (hexanes/EtOAc 15:1) afforded the title compound $( \pm)$-S5 (0.5229 g, $1.47 \mathrm{mmol}, 37 \%)$ as a colourless oil. ${ }^{1} \mathrm{H}$ NMR $\left(400 \mathrm{MHz}, \mathrm{CDCl}_{3}\right): \delta$ 7.68-7.67 $(4 \mathrm{H}, \mathrm{m}), 7.44-7.37(6 \mathrm{H}, \mathrm{m}), 5.88-5.81(1 \mathrm{H}, \mathrm{m})$, 5.13-5.08 (2H, m), 3.96-3.83 (3H, m), 3.18 (1H, br s), 2.29-2.25 (2H, m), 1.74-1.68 $(2 \mathrm{H}, \mathrm{m}), 1.05(9 \mathrm{H}, \mathrm{s})$.

The spectroscopic data were in agreement with that reported in the literature. ${ }^{10}$
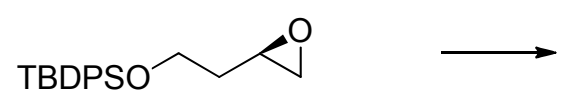

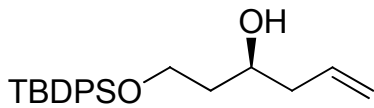

$(R)-\mathrm{S} 5$

\section{(S)-1-((tert-Butyldiphenylsilyl)oxy)hex-5-en-3-ol ((R)-S5)}

Epoxide $(R)$-S4 $(50 \mathrm{mg}, 0.15 \mathrm{mmol})$ was subjected to the general homologation procedure. Purification by column chromatography (hexanes/EtOAc 15:1) afforded the title compound $(R)$-S5 (29 mg, $0.083 \mathrm{mmol}, 51 \%, 90 \%$ e.e.) as a colourless oil. $[\alpha]_{\mathrm{D}}^{25}=-4.4\left(c 0.64\right.$ in $\left.\mathrm{CHCl}_{3}\right)$; Lit. $[\alpha]_{\mathrm{D}}^{25}=-5.4\left(c 1.03\right.$ in $\left.\mathrm{CHCl}_{3}\right)$;

HPLC: column, Chiralpak IC; mobile phase, $n$-hexane/isopropanol (99.5: $0.5 \mathrm{v} / \mathrm{v})$; flow rate, $0.5 \mathrm{~mL} / \mathrm{min}$; retention times, $18.7 \mathrm{~min}(S), 21.7 \mathrm{~min}(R)$.

The spectroscopic data were in agreement with that reported in the literature. ${ }^{10}$ 


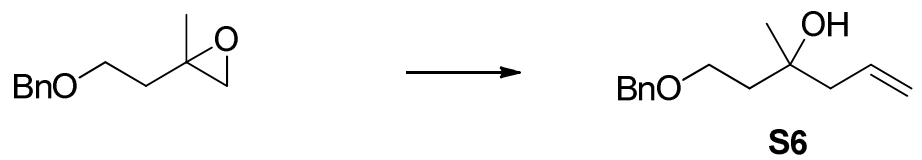

( \pm )-1-(Benzyloxy)-3-methylhex-5-en-3-ol (S6)

Epoxide $^{11}(50 \mathrm{mg}, 0.26 \mathrm{mmol})$ was subjected to the general homologation procedure. Purification by column chromatography (hexanes/EtOAc 5:1) afforded the title compound S6 (45 mg, $0.20 \mathrm{mmol}, 81 \%$ ) as a colourless oil. ${ }^{1} \mathrm{H}$ NMR (400 MHz, $\left.\mathrm{CDCl}_{3}\right): \delta 7.35-7.28(5 \mathrm{H}, \mathrm{m}), 5.90-5.80(1 \mathrm{H}, \mathrm{m}), 5.10-5.04(2 \mathrm{H}, \mathrm{m}), 4.52(2 \mathrm{H}, \mathrm{s}), 3.71$ $(2 \mathrm{H}, \mathrm{t}, J=6.0 \mathrm{~Hz}), 2.28-2.25(2 \mathrm{H}, \mathrm{m}), 1.87-1.71(2 \mathrm{H}, \mathrm{m}, \mathrm{H}-2), 1.19(3 \mathrm{H}, \mathrm{s}) ;{ }^{13} \mathrm{C} \mathrm{NMR}$ $\left(100 \mathrm{MHz}, \mathrm{CDCl}_{3}\right): \delta 137.7,134.3,128.4(\mathrm{CH} \times 2), 127.8,127.7(\mathrm{CH} \times 2), 118.0,73.4$, 72.1, 67.3, 46.9, 39.5, 26.6; IR (film) $v_{\max }$ 3443, 2972, 2925, 1720, 1454, 1364, 1093, 912, 733, $697 \mathrm{~cm}^{-1}$; HRMS (ESI+) for $\mathrm{C}_{14} \mathrm{H}_{20} \mathrm{NaO}_{2}[\mathrm{M}+\mathrm{Na}]^{+}$requires 243.1356 found 243.1349 . 

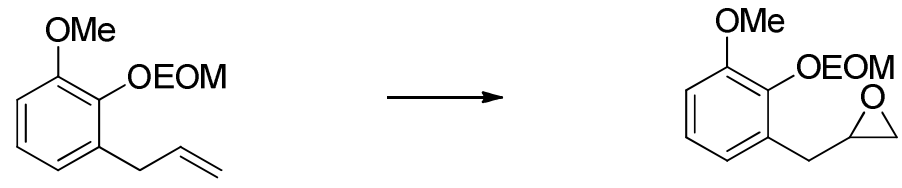

$( \pm)-$ S7

( \pm )-2-(2-(Ethoxymethoxy)-3-methoxybenzyl)oxirane $(( \pm)-S 7)$

To a stirred solution of alkene $(2.0 \mathrm{~g}, 9.00 \mathrm{mmol})$ in $\mathrm{CH}_{2} \mathrm{Cl}_{2}(45 \mathrm{~mL})$ at $0{ }^{\circ} \mathrm{C}$ was added $m$-CPBA (1.9 g, $10.8 \mathrm{mmol}, 77 \% \mathrm{wt} / \mathrm{wt})$. The reaction mixture was allowed to warm to room temperature and stirred for $2 \mathrm{~h}$. The reaction was quenched by the addition of $1 \mathrm{M}$ aq. $\mathrm{NaOH}(25 \mathrm{~mL})$ and sat. aq. $\mathrm{Na}_{2} \mathrm{SO}_{3}(25 \mathrm{~mL})$. The layers were separated and the aqueous layer further washed with water $(50 \mathrm{~mL})$, dried over anhydrous $\mathrm{MgSO}_{4}$ and concentrated in vacuo. Purification by column chromatography (hexanes/EtOAc 5:1) afforded the title compound $( \pm)-\mathrm{S} 7(1.4 \mathrm{~g}, 5.88 \mathrm{mmol}, 65 \%)$ as a colourless oil. ${ }^{1} \mathrm{H}$ NMR (400 MHz, $\left.\mathrm{CDCl}_{3}\right): \delta 7.00(1 \mathrm{H}, \mathrm{dd}, J=7.6,7.6 \mathrm{~Hz}), 6.88-6.81(2 \mathrm{H}, \mathrm{m}), 5.15$ $(2 \mathrm{H}, \mathrm{s}), 3.85-3.80(5 \mathrm{H}, \mathrm{m}), 3.22-3.19(1 \mathrm{H}, \mathrm{m}), 3.04(1 \mathrm{H}, \mathrm{dd}, J=14.4,5.6 \mathrm{~Hz}), 2.85$ $(1 \mathrm{H}, \mathrm{dd}, J=14.4,6.0 \mathrm{~Hz}), 2.78-2.76(1 \mathrm{H}, \mathrm{m}), 2.59-2.57(1 \mathrm{H}, \mathrm{m}), 1.25(3 \mathrm{H}, \mathrm{t}$, $J=7.2 \mathrm{~Hz}) ;{ }^{13} \mathrm{C} \mathrm{NMR}\left(100 \mathrm{MHz}, \mathrm{CDCl}_{3}\right): \delta 152.2,144.4,131.5,124.2,122.4,110.9$, 97.5, 65.5, 55.7, 51.9, 47.1, 33.1, 15.1; IR (film) $v_{\max } 2977,2933,1585,1474,1267$, 1210, 1070, 959, 771, $748 \mathrm{~cm}^{-1}$; HRMS (ESI+) for $\mathrm{C}_{13} \mathrm{H}_{18} \mathrm{NaO}_{4}[\mathrm{M}+\mathrm{Na}]^{+}$requires 261.1097 found 261.1097. 


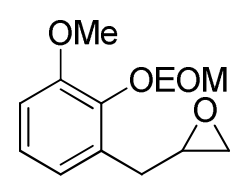

$( \pm)-S 7$

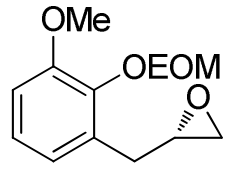

(S)-S7

\section{$(S)-2-(2-($ Ethoxymethoxy)-3-methoxybenzyl)oxirane $((S)-S 7)$}

To a stirred mixture of epoxide $( \pm)$-S7 (1.25 g, $5.25 \mathrm{mmol})$, water $(0.06 \mathrm{~mL})$ and $(S, S)$ - $N, N^{\prime}$-bis(3,5-di-tert-butylsalicylidene)-1,2-cyclohexanediaminocobalt(II) (16 mg, $0.026 \mathrm{mmol})$ at $0{ }^{\circ} \mathrm{C}$ was added acetic acid $(6 \mu \mathrm{L}, 0.11 \mathrm{mmol})$. The resultant mixture was allowed to warm to room temperature slowly overnight. Purification by column chromatography (hexanes/EtOAc 7:1) afforded the title compound (S)-S7 (0.64 g, $2.69 \mathrm{mmol}, 51 \%, 70 \%$ e.e.) as a colourless oil.

Repetition of the resolution procedure employing epoxide $(S)$-S7 (0.32 g, $1.34 \mathrm{mmol})$, afforded the title compound (S)-S7 $(0.16 \mathrm{~g}, 0.69 \mathrm{mmol}, 53 \%$, e.r. $>99: 1$ as determined by chiral HPLC) as a colourless oil. $[\alpha]_{\mathrm{D}}^{25}=+14.6\left(\right.$ c 1.2 in $\left.\mathrm{CHCl}_{3}\right)$.

HPLC: column, Chiralpak IC; mobile phase, $n$-hexane/isopropanol (65: $35 \mathrm{v} / \mathrm{v}$ ); flow rate, $0.5 \mathrm{~mL} / \mathrm{min}$; retention times, $15.2 \mathrm{~min}(R), 28.6 \mathrm{~min}(S)$. 


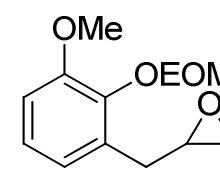

$( \pm)-\mathbf{S 7}$

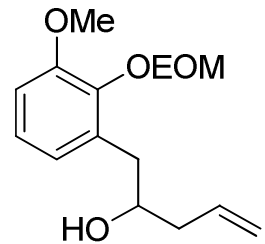

$( \pm)-\mathbf{S 8}$

( \pm )-1-(2-(Ethoxymethoxy)-3-methoxyphenyl)pent-4-en-2-ol (( \pm$)-S 8)$

Epoxide ( $( \pm)-\mathbf{S} 7(50 \mathrm{mg}, 0.21 \mathrm{mmol})$ was subjected to the general homologation procedure. Purification by column chromatography (hexanes/EtOAc 7:1) afforded the title compound $( \pm)$-S13 (51 mg, 0.19 mmol, 91\%) as a yellow oil. ${ }^{1} \mathrm{H}$ NMR (400 MHz, $\left.\mathrm{CDCl}_{3}\right): \delta 7.00(1 \mathrm{H}, \mathrm{dd}, J=8.0,8.0 \mathrm{~Hz}), 6.81-6.79(2 \mathrm{H}, \mathrm{m}), 5.94-5.84(1 \mathrm{H}, \mathrm{m})$, 5.17-5.10 (4H, m), 3.95-3.92 (1H, m), 3.85-3.80 (5H, m), 2.91-2.81 $(2 \mathrm{H}, \mathrm{m}), 2.43(1 \mathrm{H}$, s), 2.32-2.26 (2H, m), $1.25(3 \mathrm{H}, \mathrm{t}, J=6.8 \mathrm{~Hz}) ;{ }^{13} \mathrm{C} \mathrm{NMR}\left(100 \mathrm{MHz}, \mathrm{CDCl}_{3}\right): \delta 152.1$, $144.8,135.0,132.8,124.2,122.9,117.5,110.6$, 97.6, 71.4, 65.5, 55.6, 41.8, 37.6, 15.1; IR (film) $v_{\max } 3453,2976,1585,1475,1265,1208,1071,964,781,748 \mathrm{~cm}^{-1}$; HRMS $(\mathrm{ESI}+)$ for $\mathrm{C}_{15} \mathrm{H}_{22} \mathrm{NaO}_{4}[\mathrm{M}+\mathrm{Na}]^{+}$requires 289.1410 found 289.1403 .

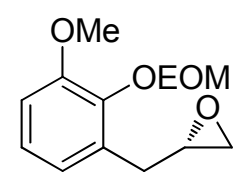

(S)-S7

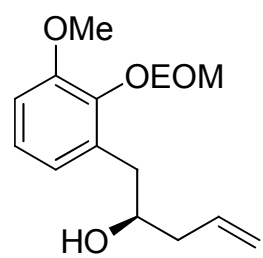

$(R)-\mathrm{S} 8$

(R)-1-(2-(Ethoxymethoxy)-3-methoxyphenyl)pent-4-en-2-ol $((R)-\mathrm{S8})$

Epoxide $(S)$-S7 $(50 \mathrm{mg}, 0.21 \mathrm{mmol})$ was subjected to the general homologation procedure. Purification by column chromatography (hexanes/EtOAc 7:1) afforded the title compound $(R)-\mathbf{S 8}(50 \mathrm{mg}, 0.19 \mathrm{mmol}, 90 \%,>99 \%$ e.e.) as a colourless oil. $[\alpha]_{\mathrm{D}}^{25}=-3.4\left(c 0.98\right.$ in $\left.\mathrm{CHCl}_{3}\right)$.

HPLC: column, Chiralpak AD-H; mobile phase, $n$-hexane/isopropanol (99 : $1 \mathrm{v} / \mathrm{v})$; flow rate, $0.5 \mathrm{~mL} / \mathrm{min}$; retention times, $102.3 \mathrm{~min}(R), 108.5 \mathrm{~min}(S)$. 



\section{( \pm )-1-(2-Methoxyphenyl)pent-4-en-2-ol (S9)}

Epoxide $^{12}(50 \mathrm{mg}, 0.21 \mathrm{mmol})$ was subjected to the general homologation procedure. Purification by column chromatography (hexanes/EtOAc 5:1) afforded the title compound S9 (43 mg, $0.16 \mathrm{mmol}, 77 \%$ ) as a colourless oil. ${ }^{1} \mathrm{H}$ NMR (400 MHz, $\left.\mathrm{CDCl}_{3}\right): \delta 7.42-7.31(5 \mathrm{H}, \mathrm{m}), 7.22-7.17(2 \mathrm{H}, \mathrm{m}), 6.93-6.90(2 \mathrm{H}, \mathrm{m}), 5.87-5.80(1 \mathrm{H}, \mathrm{m})$, 5.13-5.07 (4H, m), 3.99-3.93 $(1 \mathrm{H}, \mathrm{m}), 2.96(1 \mathrm{H}, \mathrm{dd}, J=13.6,4.4 \mathrm{~Hz}), 2.74(1 \mathrm{H}, \mathrm{dd}$, $J=13.6,8.0 \mathrm{~Hz}), 2.29-2.23(2 \mathrm{H}, \mathrm{m}) ;{ }^{13} \mathrm{C} \mathrm{NMR}\left(100 \mathrm{MHz}, \mathrm{CDCl}_{3}\right): \delta 156.7,136.9$, 135.0, 131.5, $128.5(\mathrm{CH} \times 2), 127.9,127.8,127.3,127.2(\mathrm{CH} \times 2), 120.9,117.5,111.8$, 70.9, 70.0, 41.4, 38.1; IR (film) $v_{\max } 3425,3033,2922,1600,1491,1451,1238,1022$, 912, 749, $695 \mathrm{~cm}^{-1}$; HRMS (ESI+) for $\mathrm{C}_{18} \mathrm{H}_{20} \mathrm{NaO}_{2}[\mathrm{M}+\mathrm{Na}]^{+}$requires 291.1356 found 291.1360 . 

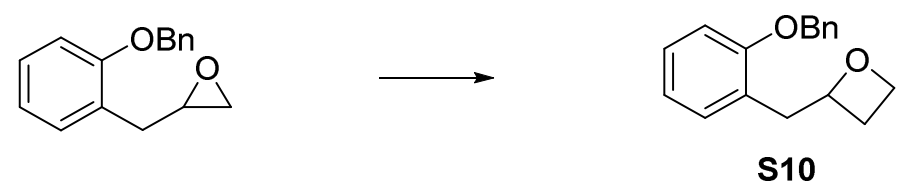

\section{2-(2-(Benzyloxy)benzyl)oxetane (S10)}

Epoxide $^{12}(50 \mathrm{mg}, 0.21 \mathrm{mmol})$ was subjected to the general homologation procedure using $\mathrm{FeCl}_{3}(2 \mathrm{mg}, 0.01 \mathrm{mmol})$ instead of catalyst B. Purification by column chromatography (petroleum ether/EtOAc 5:1) afforded the title compound $\mathbf{S 1 0}$ (53 $\mathrm{mg}$, $0.21 \mathrm{mmol}, 100 \%)$ as a colourless oil. ${ }^{1} \mathrm{H}$ NMR (400 $\left.\mathrm{MHz}, \mathrm{CDCl}_{3}\right): \delta 7.42-7.33(5 \mathrm{H}$, m), 7.22-7.17 (2H, m), 6.93-6.90 (2H, m), 5.13-5.08 $(3 \mathrm{H}, \mathrm{m}), 4.66-4.61(1 \mathrm{H}, \mathrm{m})$, 4.53-4.48 (1H, m), 3.15 (2H, ABX, $J=13.6,7.2,6.4 \mathrm{~Hz}), 2.65-2.57(1 \mathrm{H}, \mathrm{m}), 2.49-2.41$ $(1 \mathrm{H}, \mathrm{m}) ;{ }^{13} \mathrm{C} \mathrm{NMR}\left(100 \mathrm{MHz}, \mathrm{CDCl}_{3}\right): \delta 156.7,137.3,130.9,128.5(\mathrm{CH} \times 2), 127.8$, $127.7,127.1(\mathrm{CH} \times 2), 125.7,120.7,111.6,81.8,69.9,68.0,38.4,27.4$; IR (film) $v_{\max }$ $2878,1601,1493,1452,1240,1124,975 \mathrm{~cm}^{-1}$; HRMS (ESI+) for $\mathrm{C}_{17} \mathrm{H}_{18} \mathrm{NaO}_{2}[\mathrm{M}+\mathrm{Na}]^{+}$ requires 277.1199 found 277.1195 .


\section{1-(tert-Butyl)but-3-en-1-ol (S11)}

3,3-Dimethyl-1,2-epoxybutane (100 $\mathrm{mg}, 1.00 \mathrm{mmol})$ was subjected to the general homologation procedure. Purification by column chromatography (n-pentane/Et ${ }_{2} \mathrm{O} 10: 1$ ) afforded the title compound S11 (56 mg, $0.44 \mathrm{mmol}, 44 \%$ ) as a colourless oil. ${ }^{1} \mathrm{H}$ NMR $\left(400 \mathrm{MHz}, \mathrm{CDCl}_{3}\right): \delta$ 5.92-5.82 $(1 \mathrm{H}, \mathrm{m}), 5.17-5.12(2 \mathrm{H}, \mathrm{m}), 3.27-3.24(1 \mathrm{H}, \mathrm{m})$, 2.40-2.34 $(1 \mathrm{H}, \mathrm{m}), 2.04-1.94(1 \mathrm{H}, \mathrm{m}), 0.92(9 \mathrm{H}, \mathrm{m})$; Due to the volatile nature of alkene S11, solvent was not completely removed following purification and the yield determined by the ${ }^{1} \mathrm{H}$ NMR peak ratios.

The spectroscopic data were in agreement with that reported in the literature. ${ }^{13}$ 

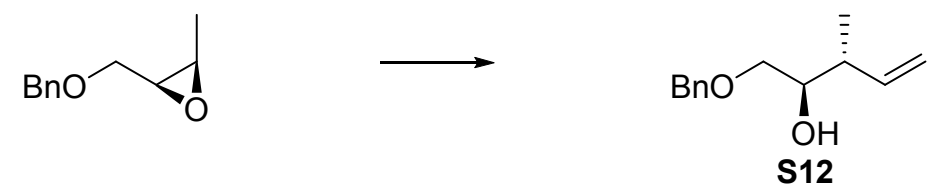

\section{(2R)-1-(Benzyloxy)-3-methylpent-4-en-2-ol (S12)}

Epoxide $^{14}(50 \mathrm{mg}, 0.28 \mathrm{mmol})$ was subjected to the general homologation procedure. Purification by column chromatography (hexanes/EtOAc 10:1) afforded the title compound S12 (20 mg, $0.097 \mathrm{mmol}, 35 \%)$ as a colourless oil. $[\alpha]_{\mathrm{D}}^{25}=+11.8(c 0.08$ in $\left.\mathrm{CHCl}_{3}\right) ;{ }^{1} \mathrm{H} \mathrm{NMR}\left(400 \mathrm{MHz}, \mathrm{CDCl}_{3}\right): \delta 7.36-7.29(5 \mathrm{H}, \mathrm{m}), 5.87-5.78(1 \mathrm{H}, \mathrm{m})$, 5.11-5.06 (1H, m), $4.56(2 \mathrm{H}, \mathrm{s}), 3.69-3.66(1 \mathrm{H}, \mathrm{m}), 3.56-3.53(1 \mathrm{H}, \mathrm{m}), 3.45-3.40(1 \mathrm{H}$, m), 2.38-2.33 (1H, m), $2.26(1 \mathrm{H}, \mathrm{d}, J=2.8 \mathrm{~Hz}), 1.04(1 \mathrm{H}, \mathrm{d}, J=6.8 \mathrm{~Hz})$.

The spectroscopic data were in agreement with that reported in the literature. ${ }^{15}$<smiles>COc1cccc(C=O)c1OCc1ccccc1</smiles>



\section{( \pm )-1-(2-(Benzyloxy)-3-methoxyphenyl)but-3-en-1-ol (S13)}

2-Benzyloxy-3-methoxybenzaldehyde (50 mg, $0.21 \mathrm{mmol}$ ) was subjected to the general homologation procedure. Purification by column chromatography (hexanes/EtOAc 10:1) afforded the title compound $\mathbf{S 2 2}(25 \mathrm{mg}, 0.088 \mathrm{mmol}, 43 \%$ ) as a colourless oil. ${ }^{1} \mathrm{H}$ NMR (400 MHz, $\left.\mathrm{CDCl}_{3}\right): \delta 7.44-7.32(5 \mathrm{H}, \mathrm{m}), 7.08(1 \mathrm{H}, \mathrm{dd}, J=8.0,8.0 \mathrm{~Hz}), 7.00-$ $6.98(1 \mathrm{H}, \mathrm{m}), 6.89-6.87(1 \mathrm{H}, \mathrm{m}), 5.80-5.72(1 \mathrm{H}, \mathrm{m}), 5.12-5.02(4 \mathrm{H}, \mathrm{m}) 4.96-4.93(1 \mathrm{H}$, m), $3.90(3 \mathrm{H}, \mathrm{s}), 2.46-2.42(2 \mathrm{H}, \mathrm{m}) ;{ }^{13} \mathrm{C} \mathrm{NMR}\left(100 \mathrm{MHz}, \mathrm{CDCl}_{3}\right): \delta 152.4,144.6$, $137.7,137.6,134.9,128.4(\mathrm{CH} \times 2), 128.2(\mathrm{CH} \times 2), 128.1,124.2,118.5,117.8,111.5$, 74.8, 68.5, 55.8, 42.4; IR (film) $v_{\max } 3423,2936,1585,1476,1269,1202,1048,988$, 913, $748 \mathrm{~cm}^{-1}$; HRMS (ESI+) for $\mathrm{C}_{18} \mathrm{H}_{20} \mathrm{NaO}_{3}[\mathrm{M}+\mathrm{Na}]^{+}$requires 307.1313 found 307.1305 . 


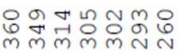

min

$+1$

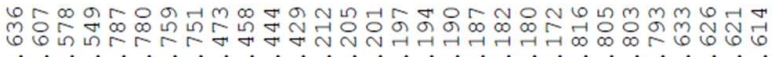

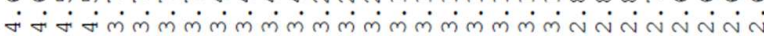

r.
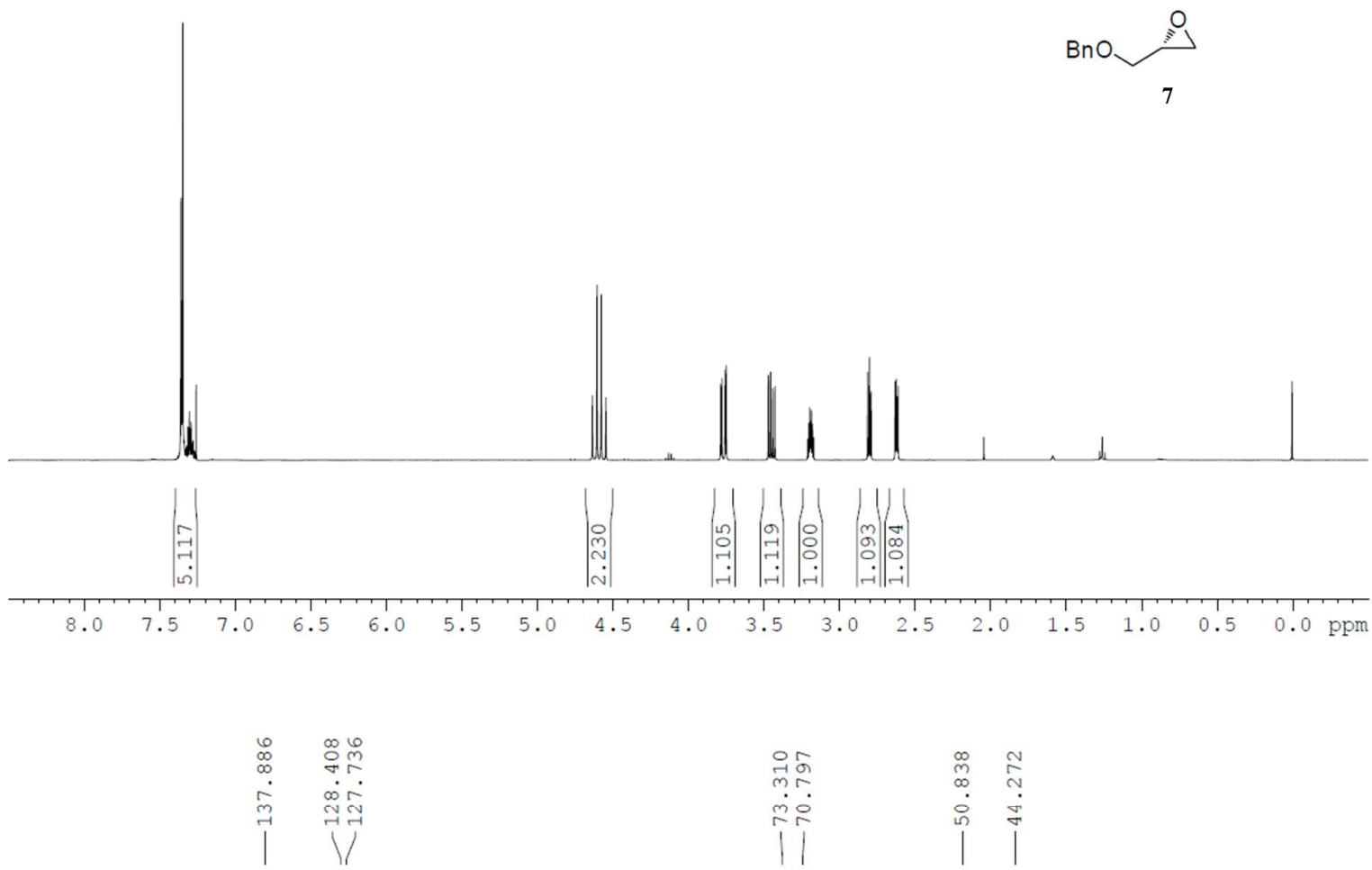

|l

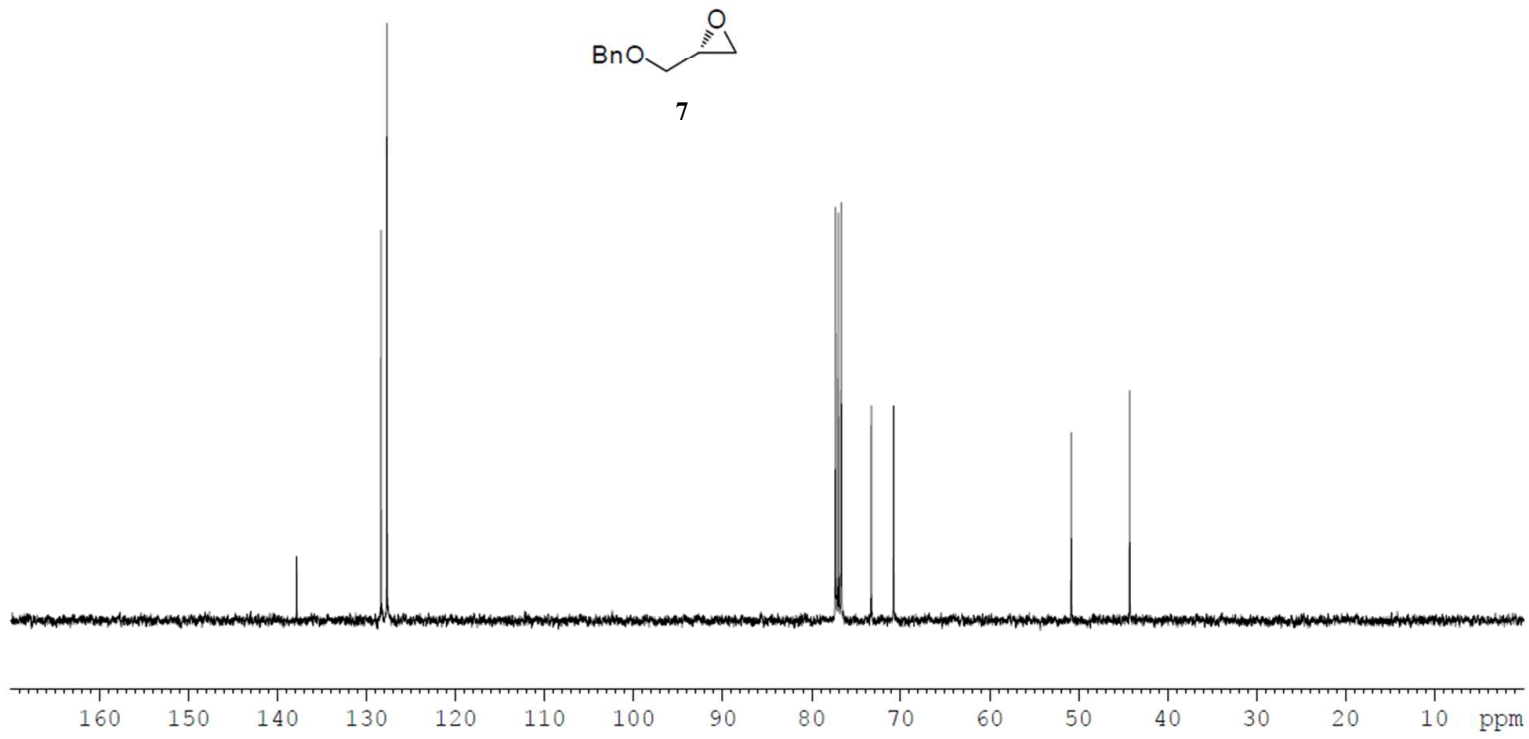






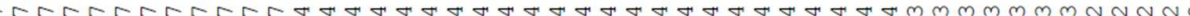
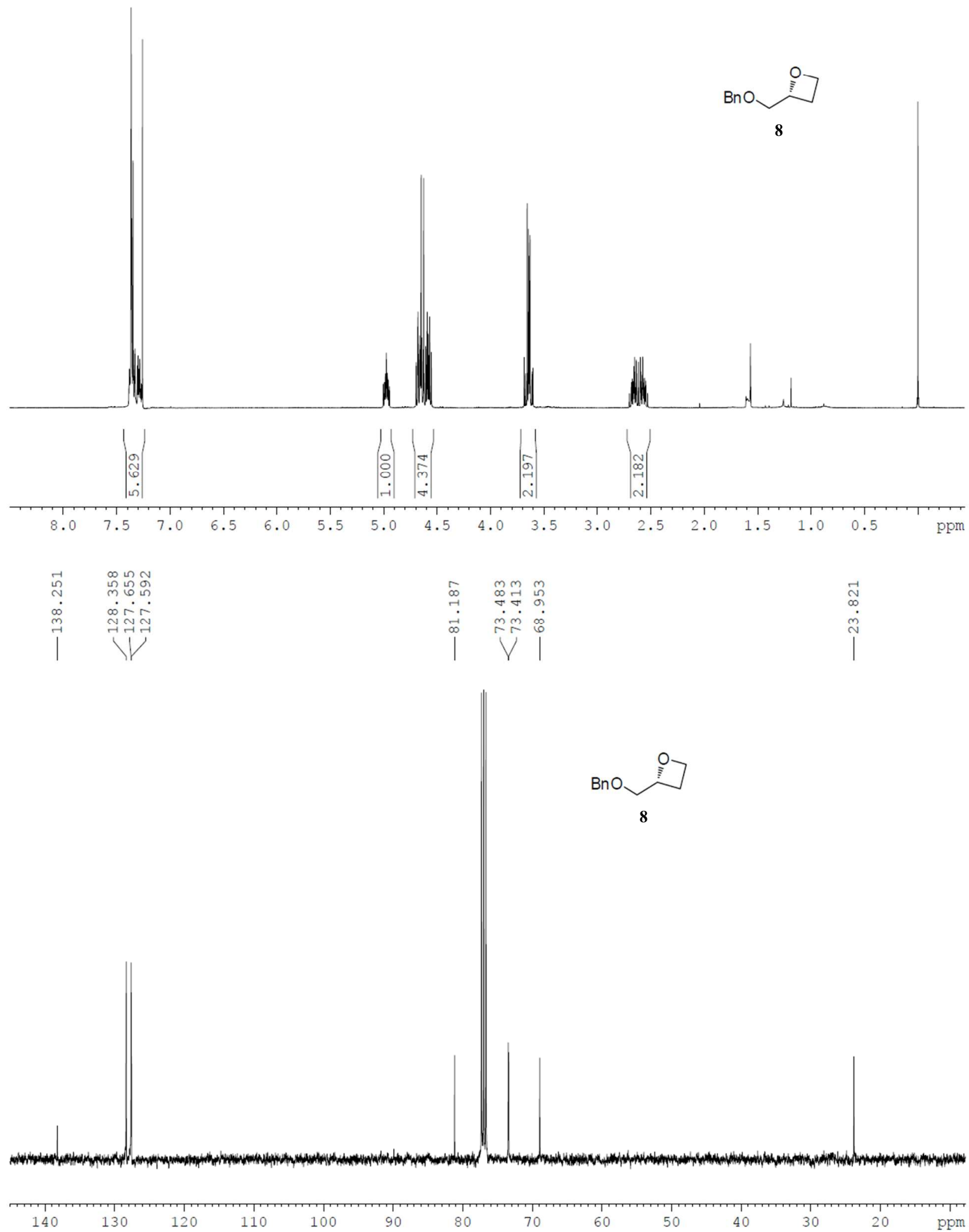


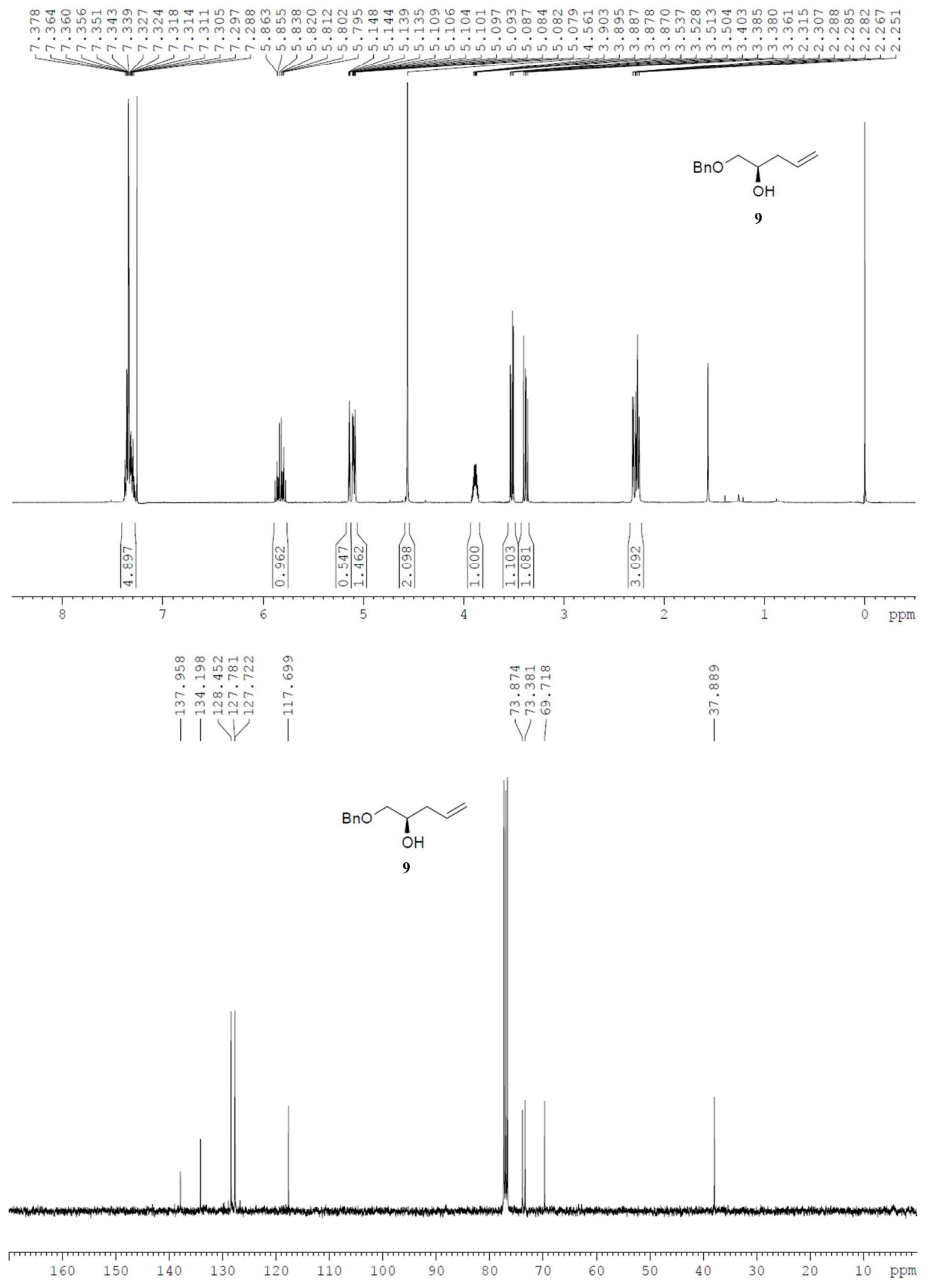




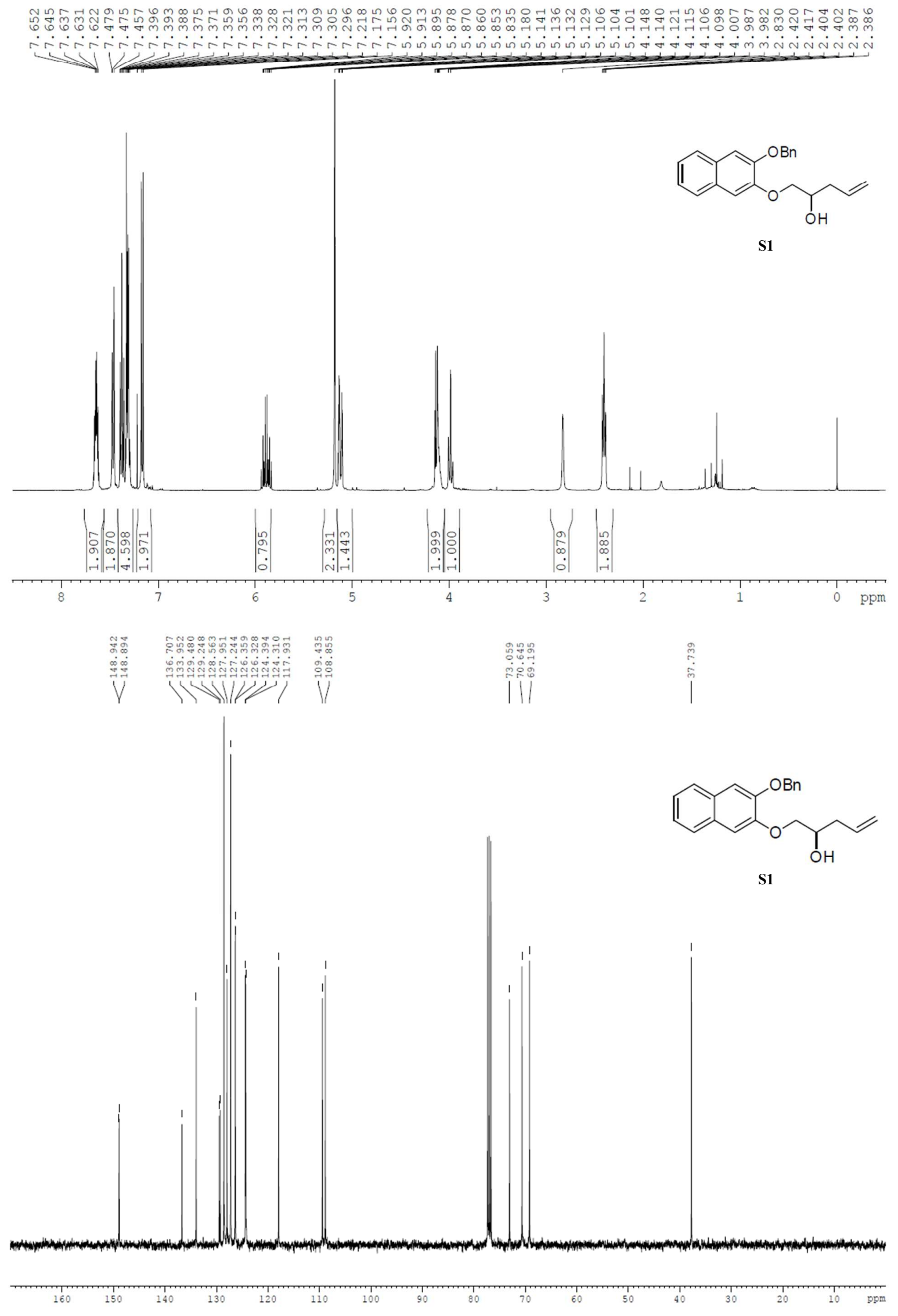





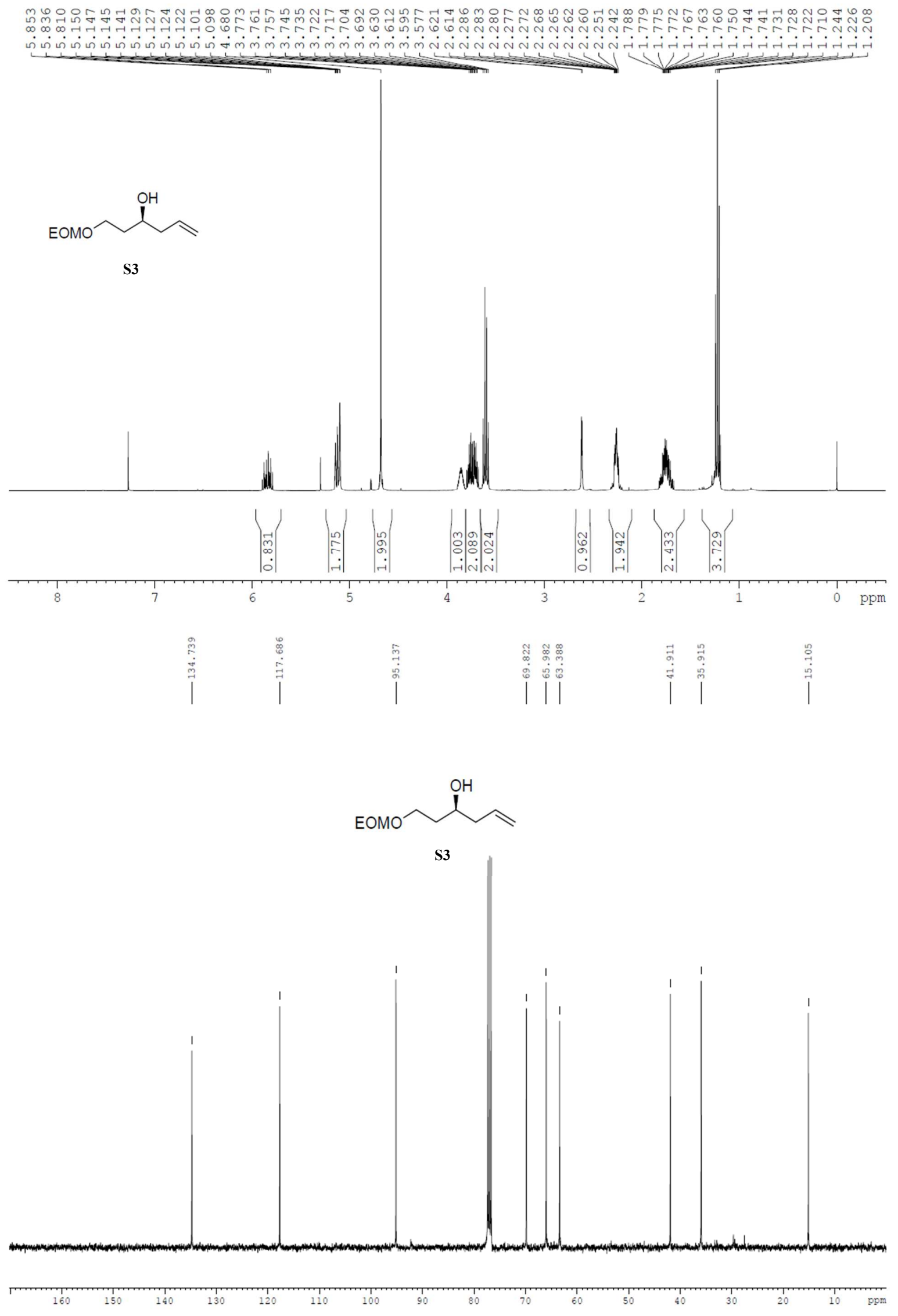

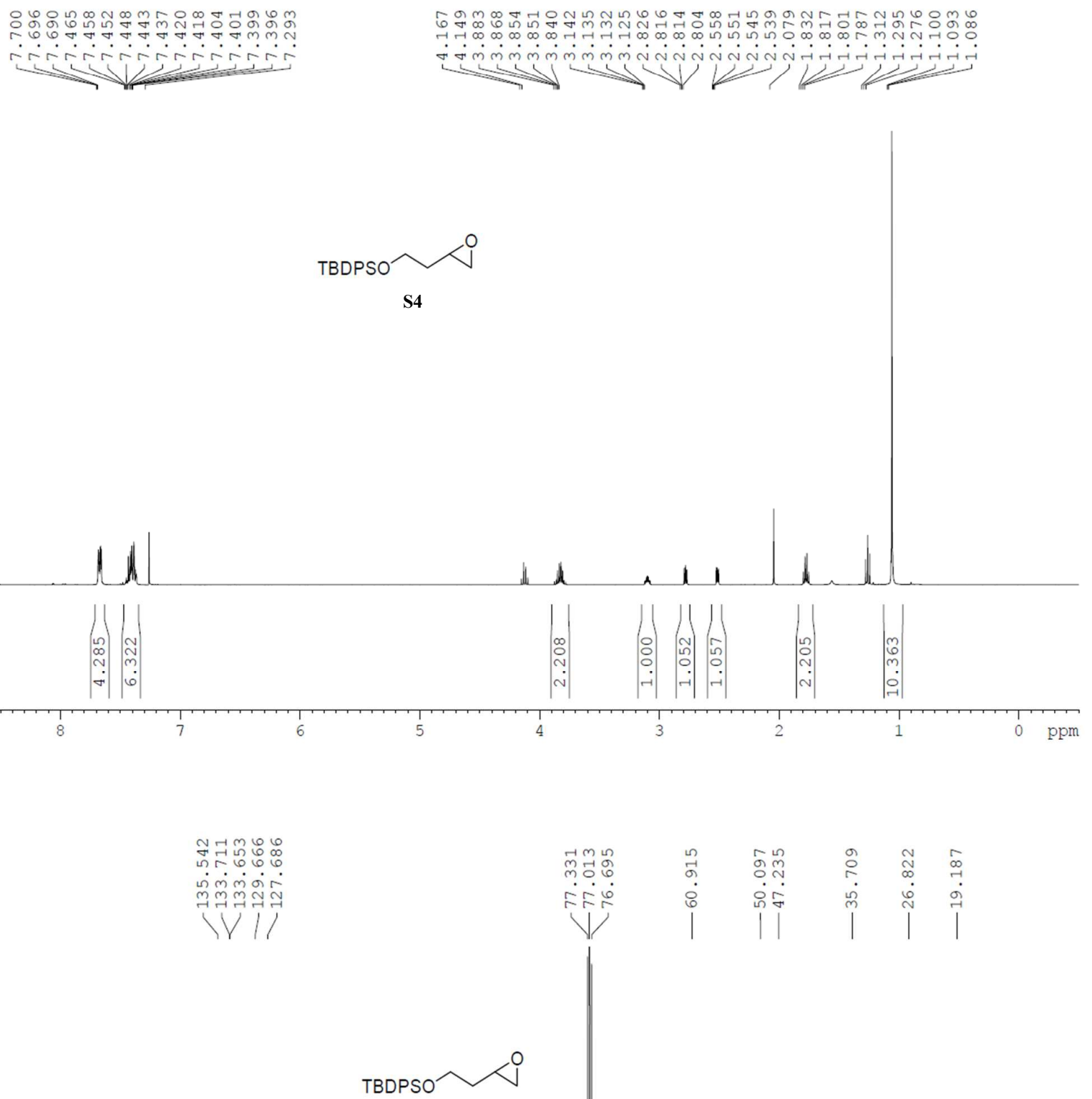

S4


60

$50 \quad 40$

$2010 \mathrm{ppm}$ 


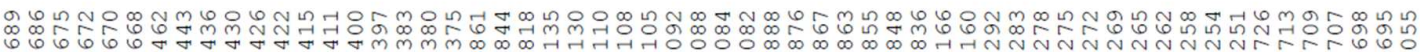

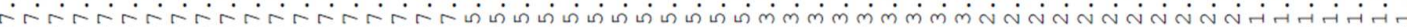

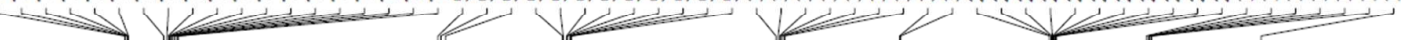



S5

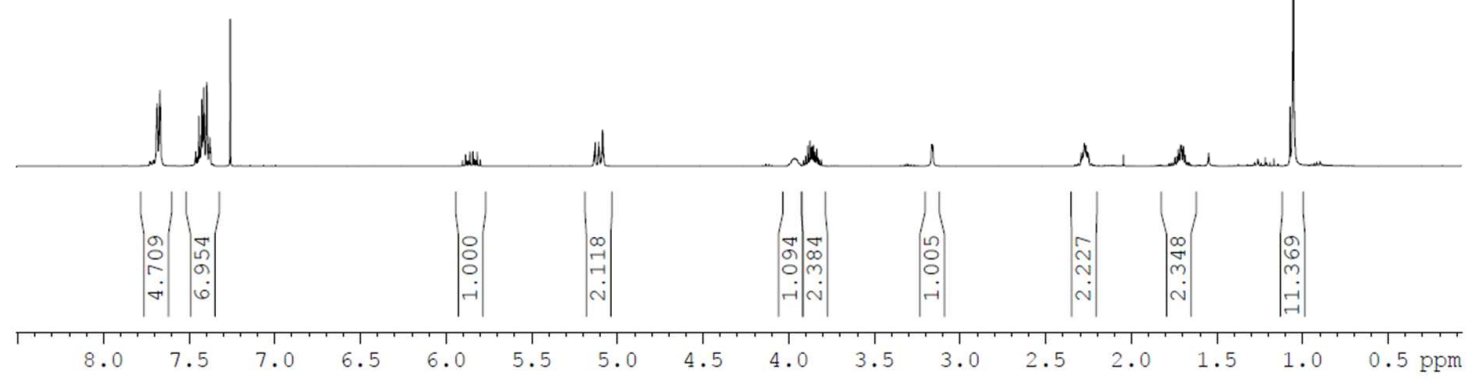

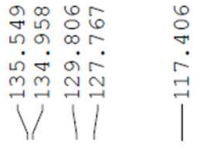
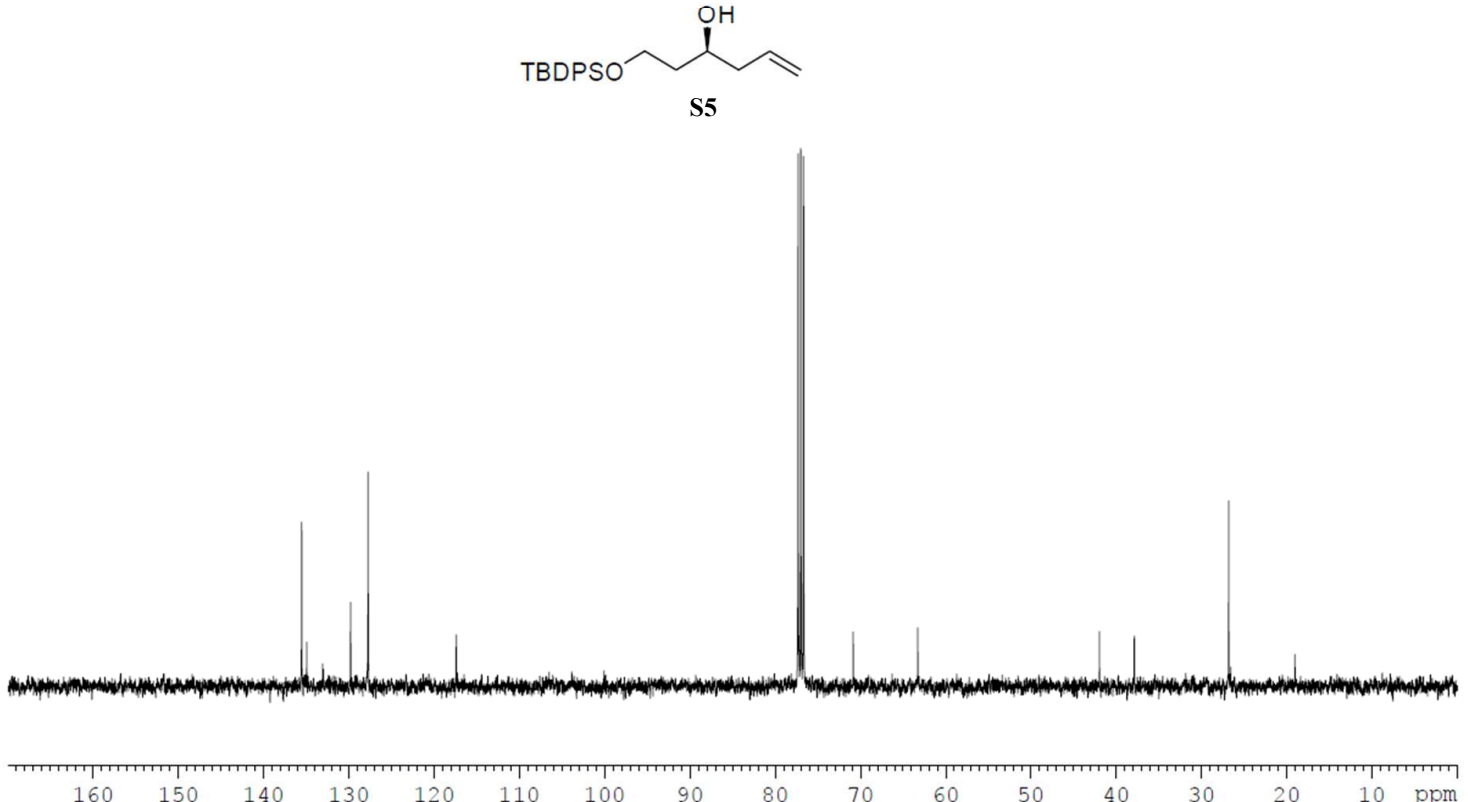


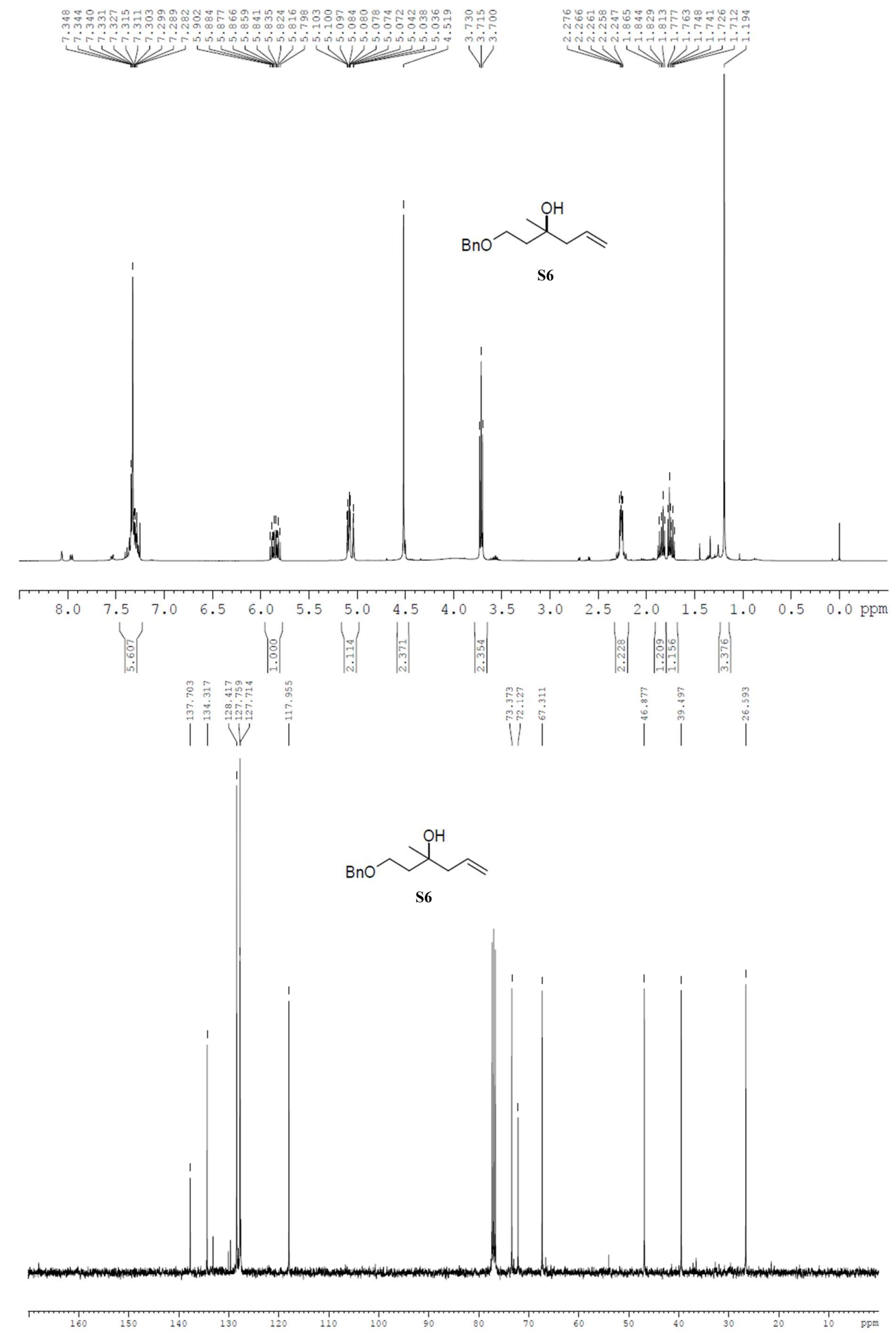



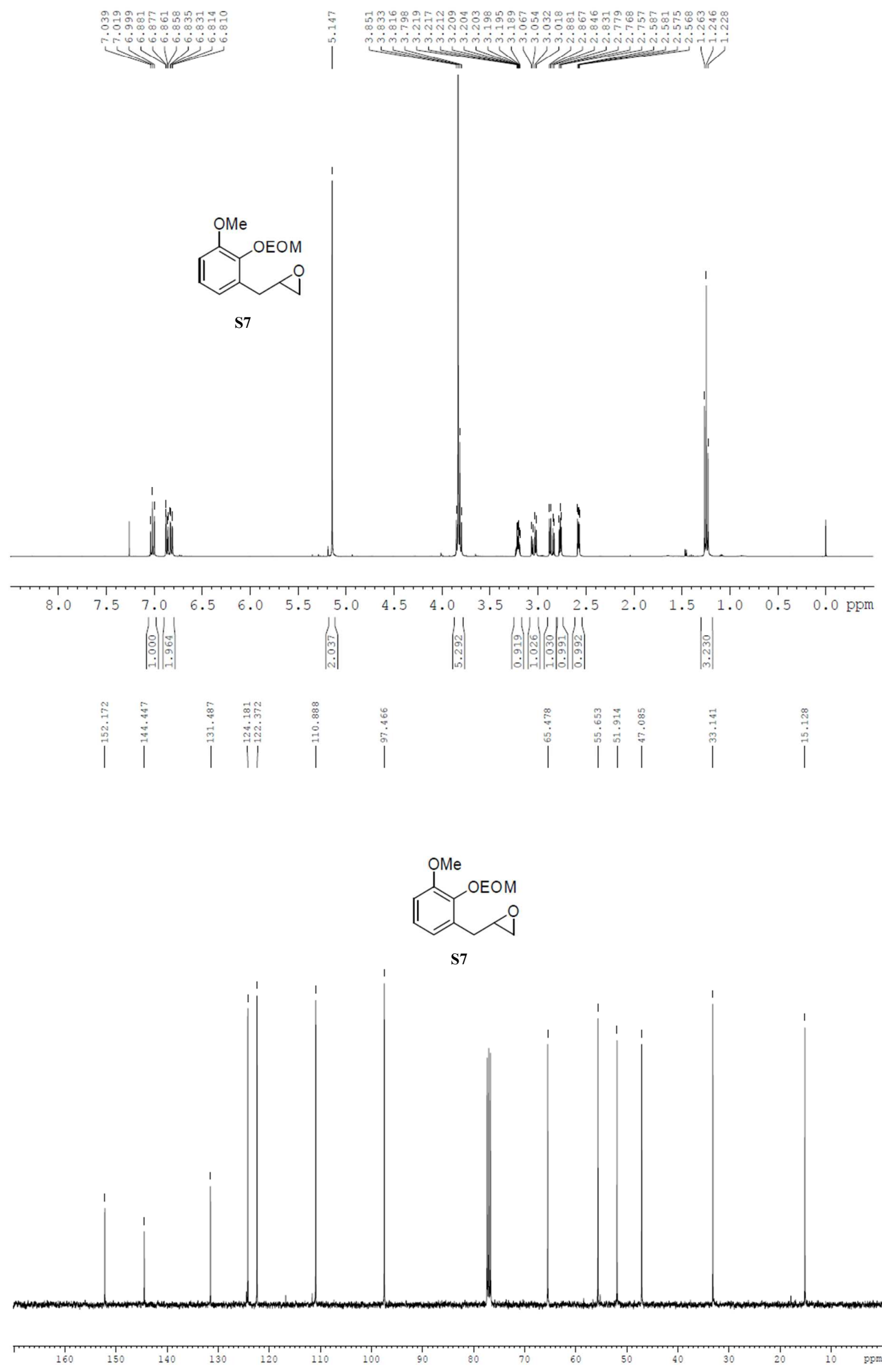


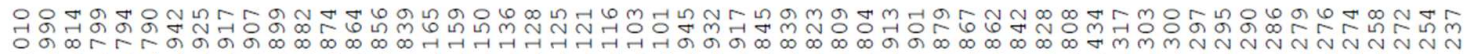

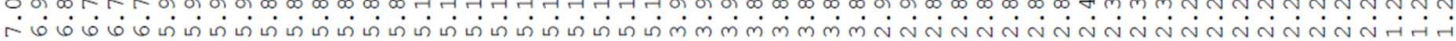

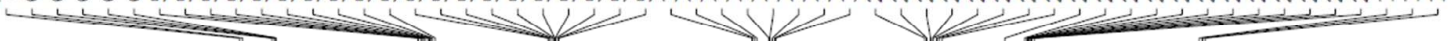

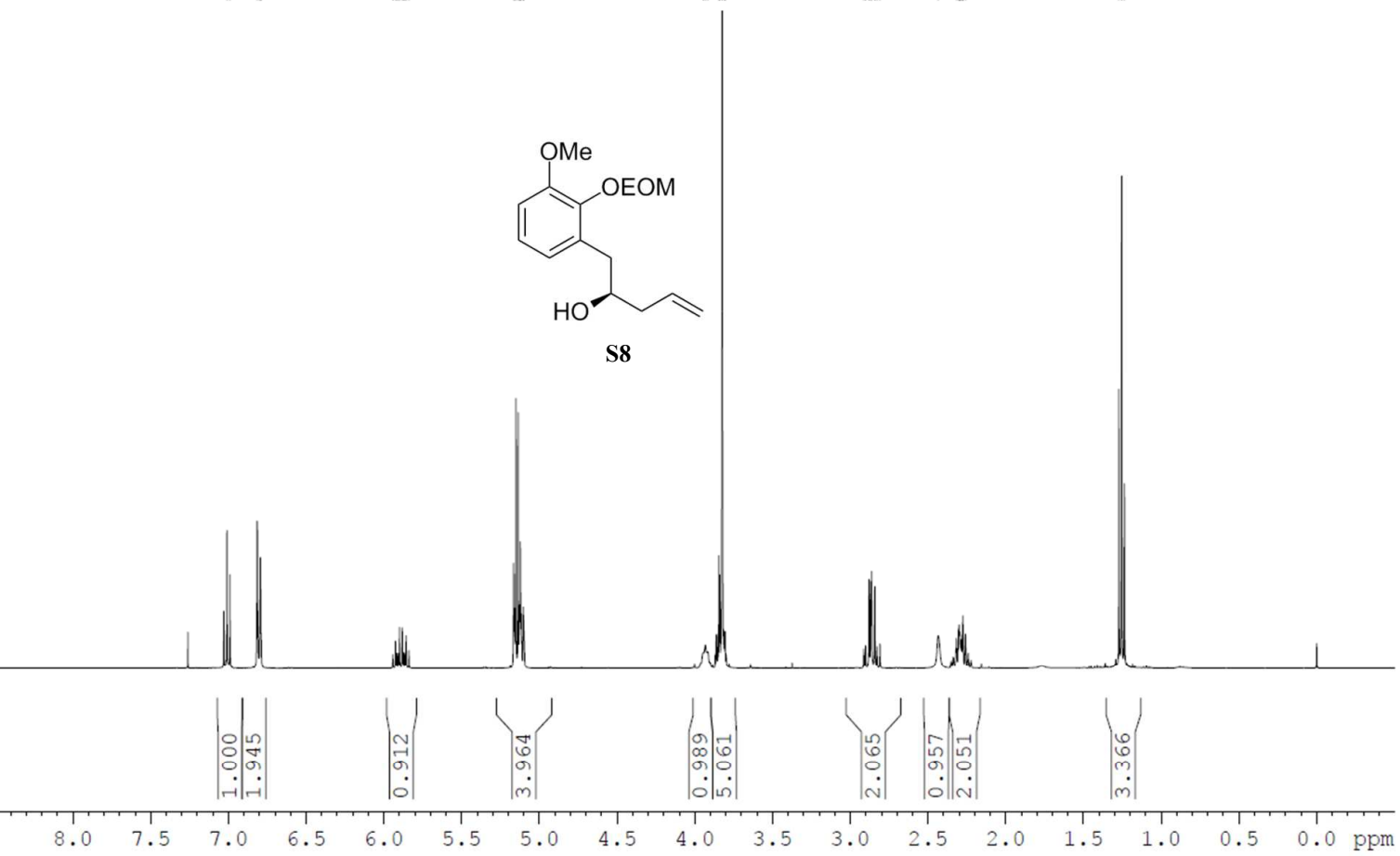

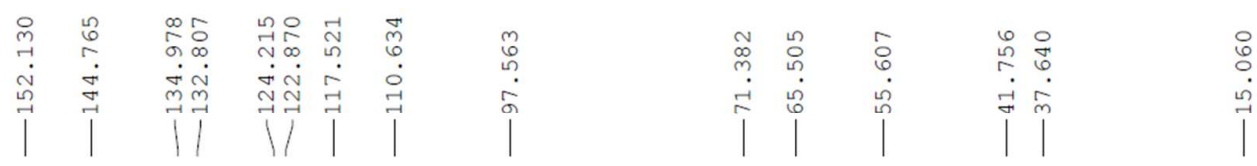<smiles>C=CC[C@H](O)Cc1cccc(OC)c1OCC</smiles>

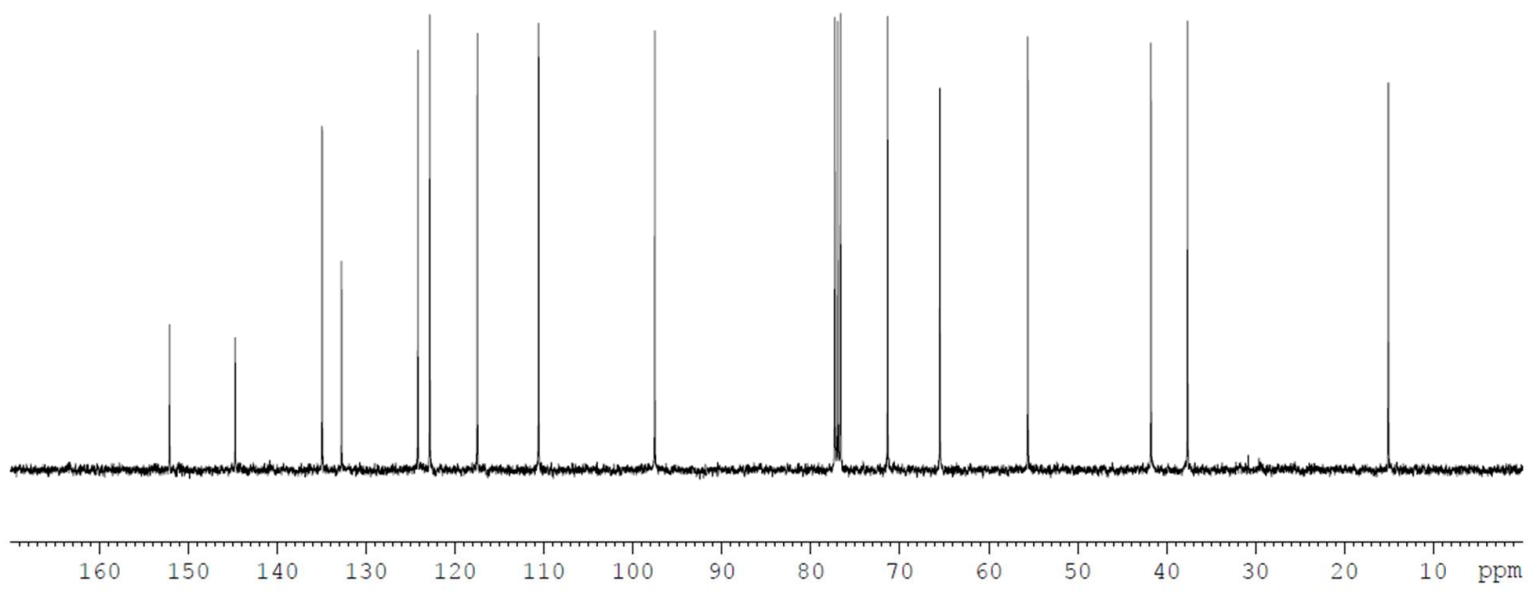




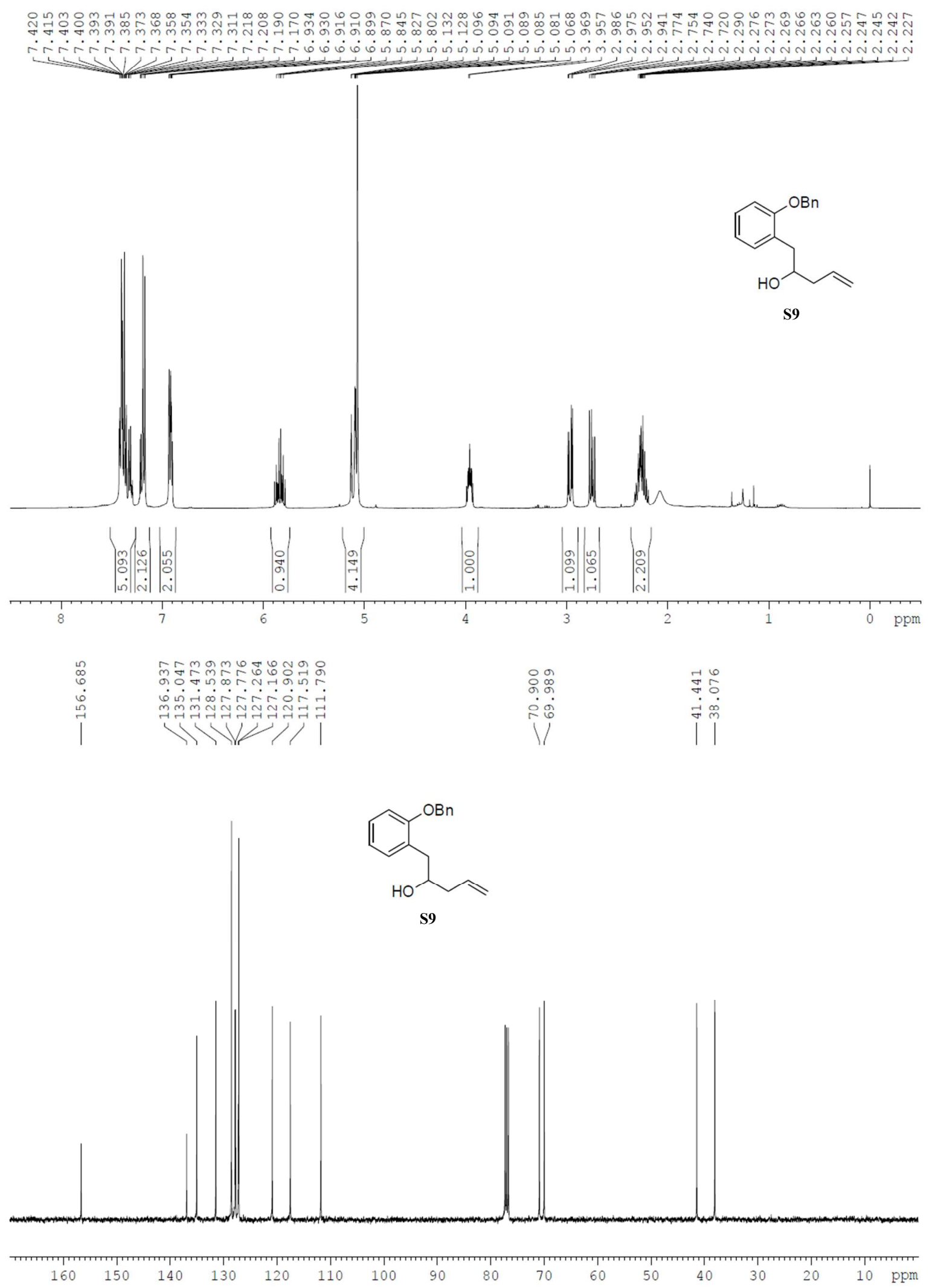



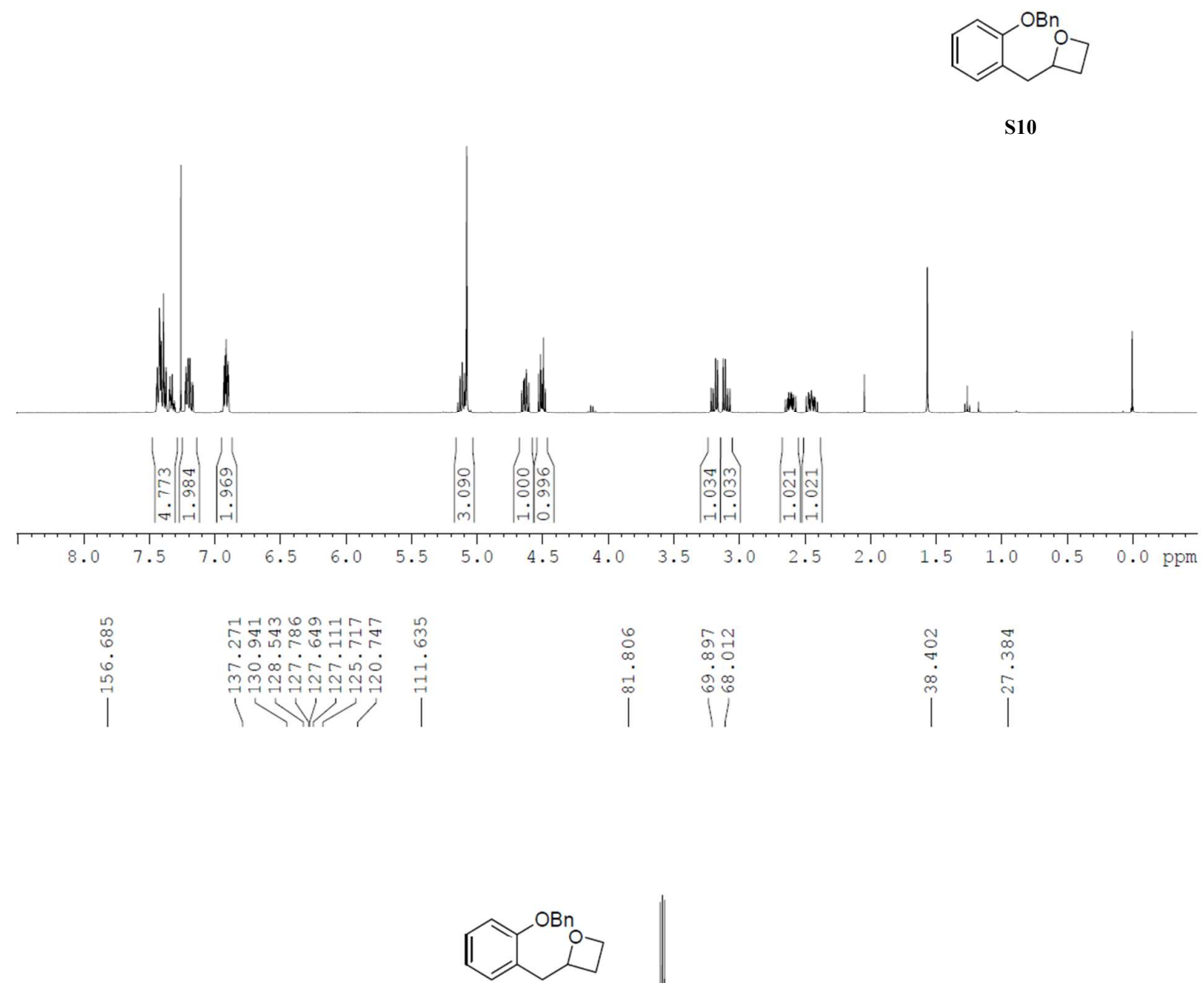

S10
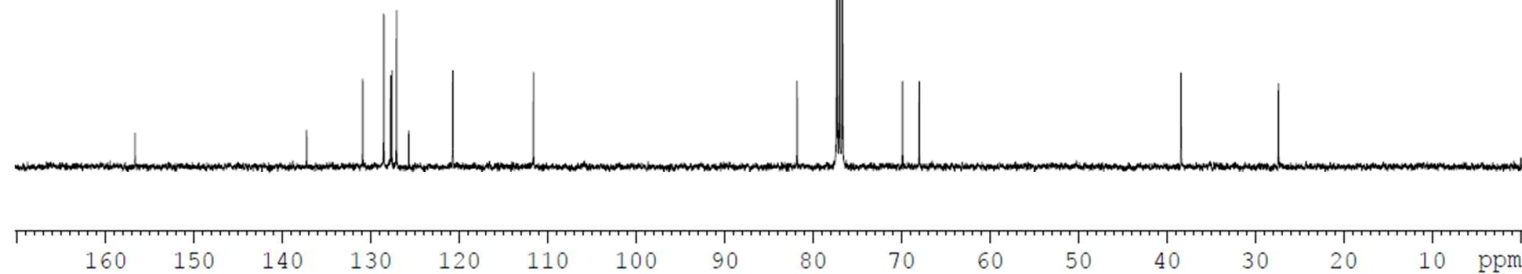

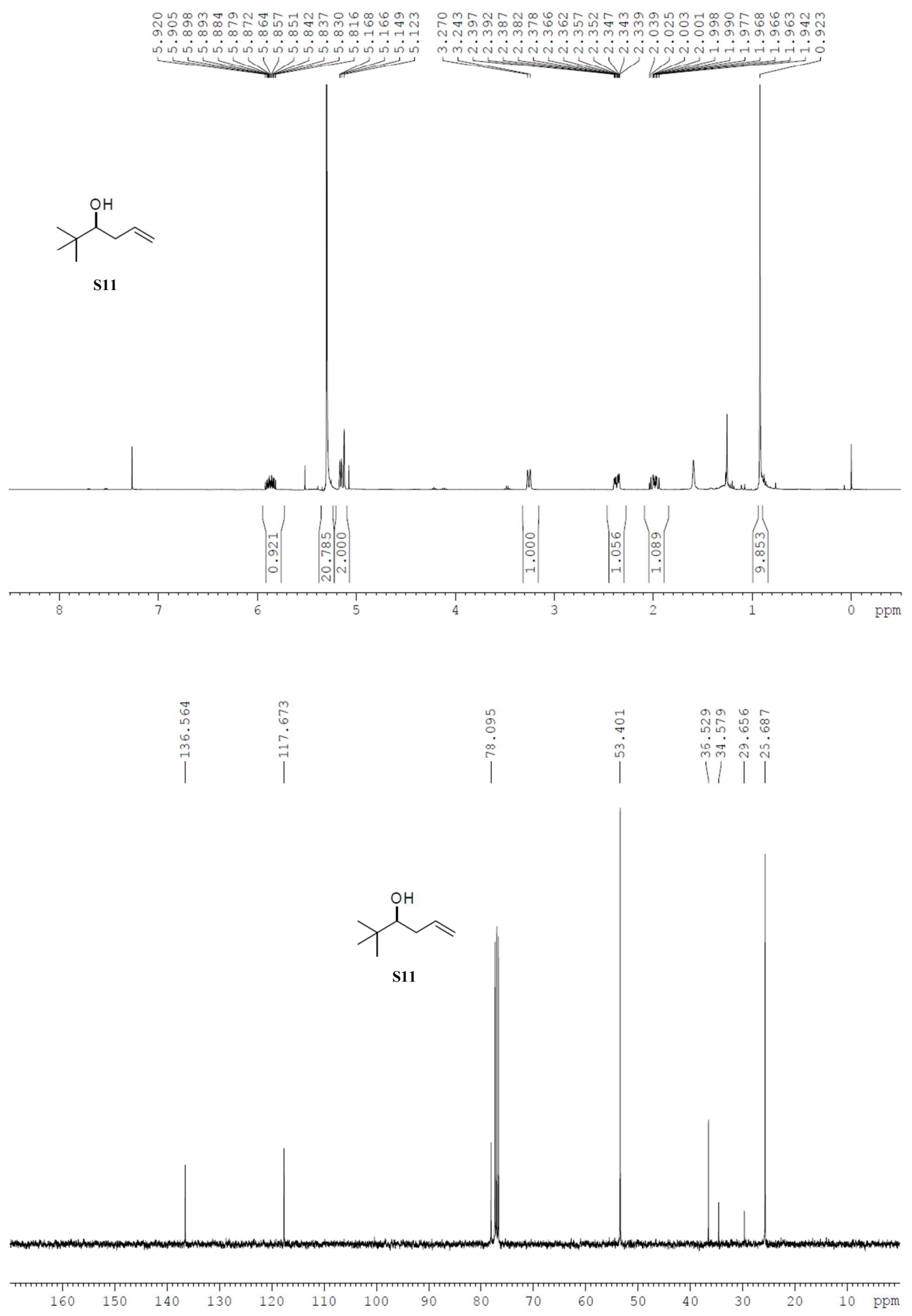




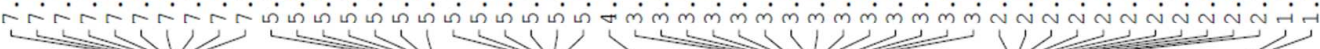
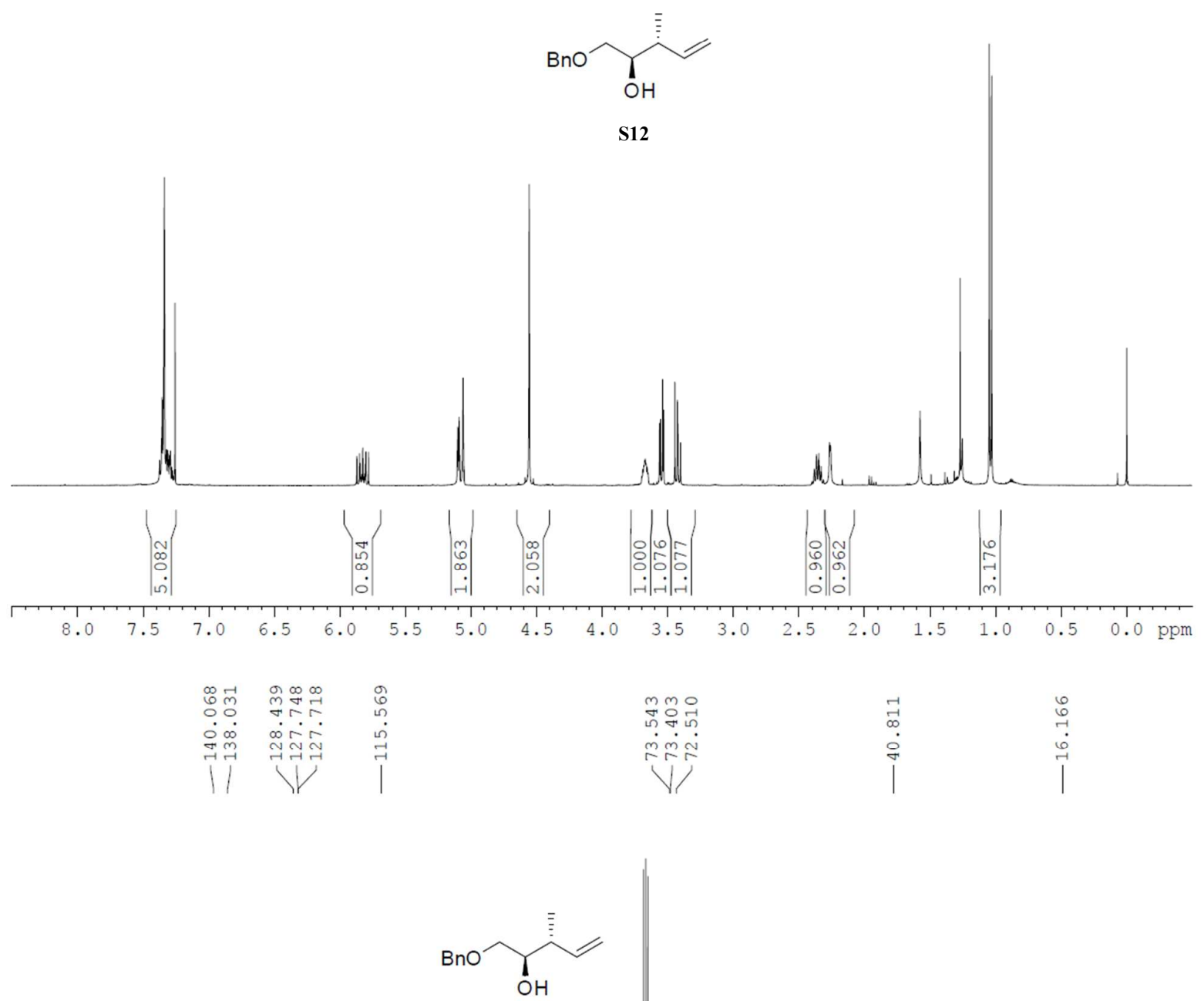

S12
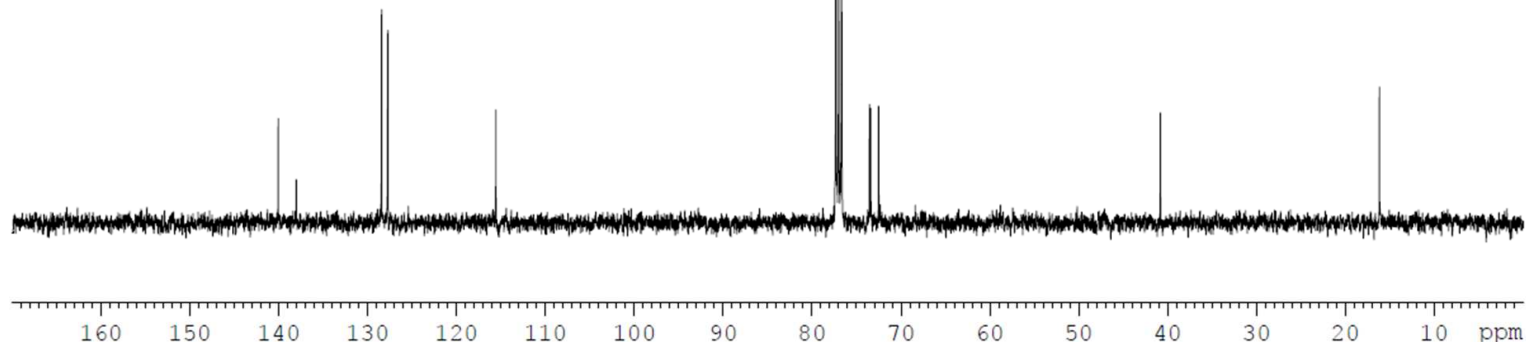

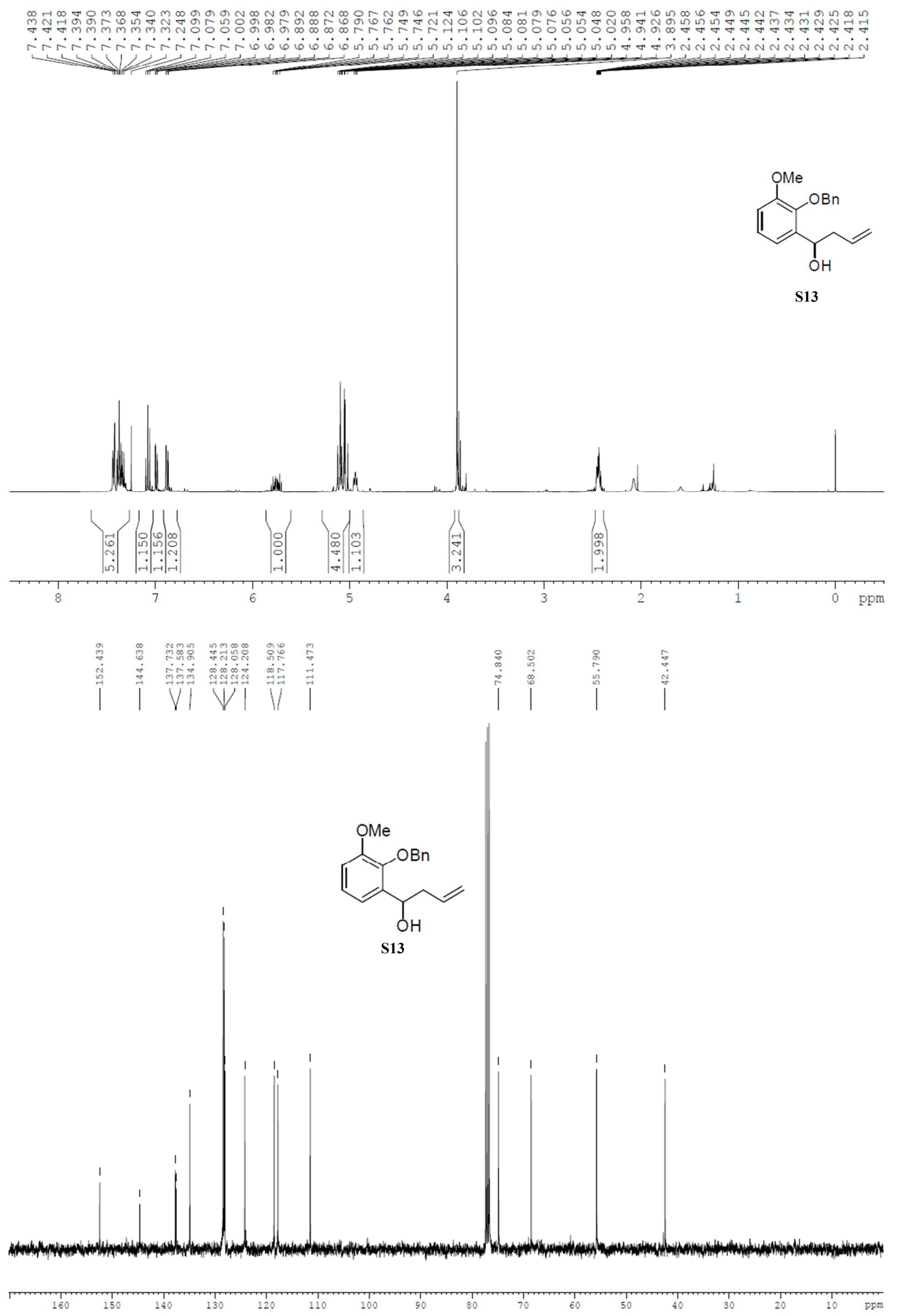
${ }^{19}$ F NMR spectra of Mosher ester derivatives of 9
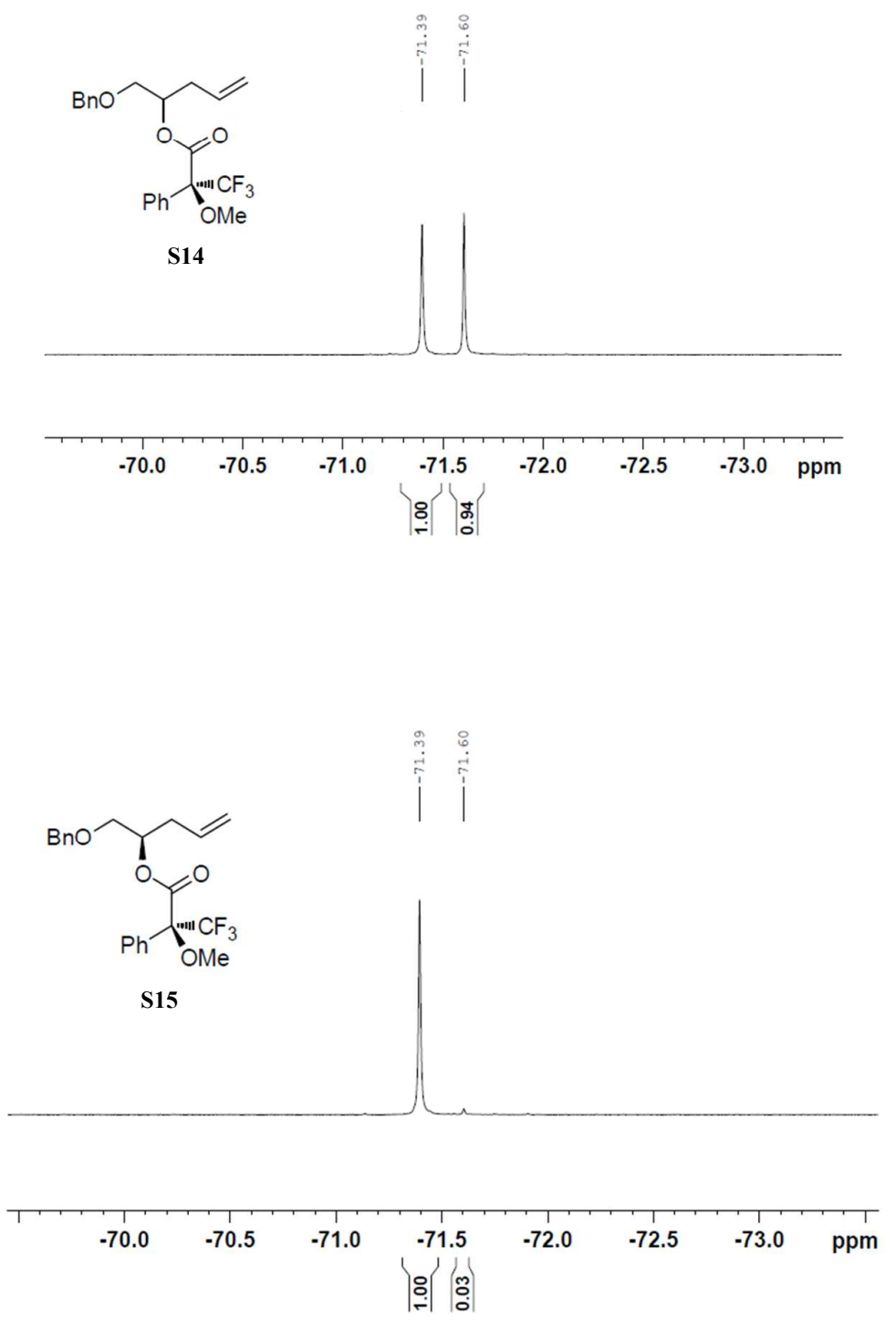


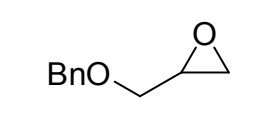

(土)-7

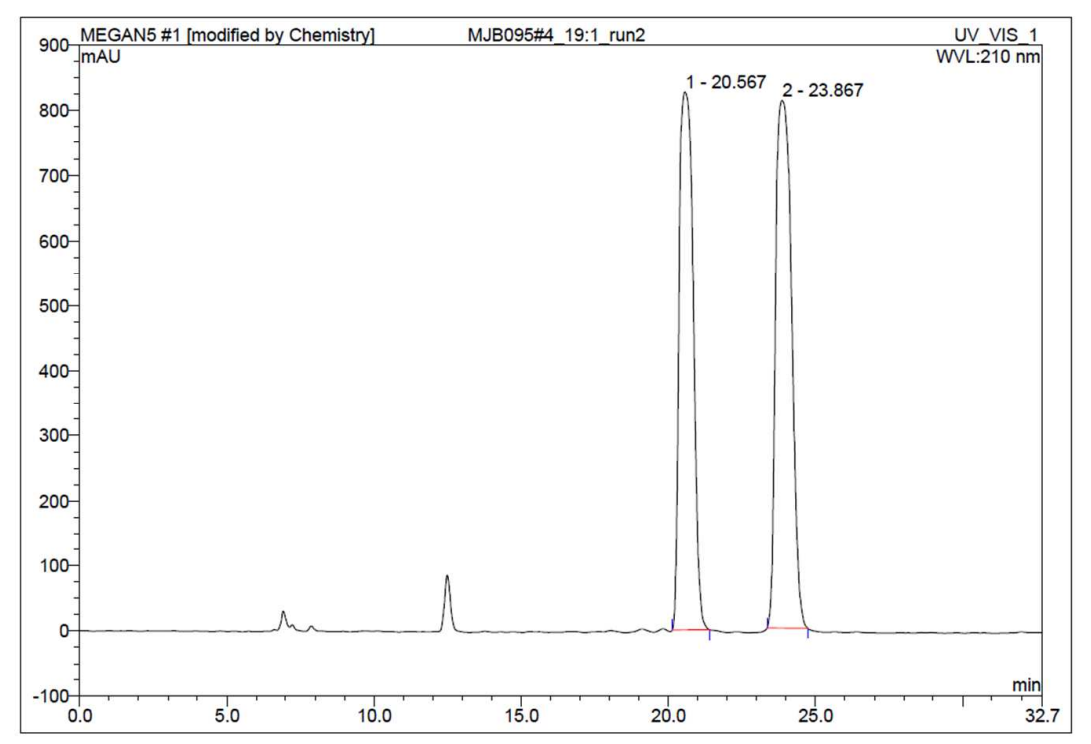

\begin{tabular}{|r|cccrrrr|}
\hline No. & $\begin{array}{c}\text { Ret.Time } \\
\text { min }\end{array}$ & Peak Name & $\begin{array}{c}\text { Height } \\
\text { mAU }\end{array}$ & $\begin{array}{c}\text { Area } \\
\mathrm{mAU}^{*} \text { min }\end{array}$ & $\begin{array}{c}\text { Rel.Area } \\
\%\end{array}$ & Amount & Type \\
\hline 1 & 20.57 & n.a. & 826.668 & 454.856 & 47.31 & n.a. & BMB $^{*}$ \\
2 & 23.87 & n.a. & 811.367 & 506.605 & 52.69 & n.a. & BMB $^{*}$ \\
\hline Total: & & & 1638.035 & 961.461 & 100.00 & 0.000 & \\
\hline
\end{tabular}

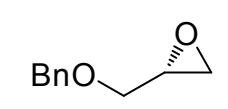

(R)-7

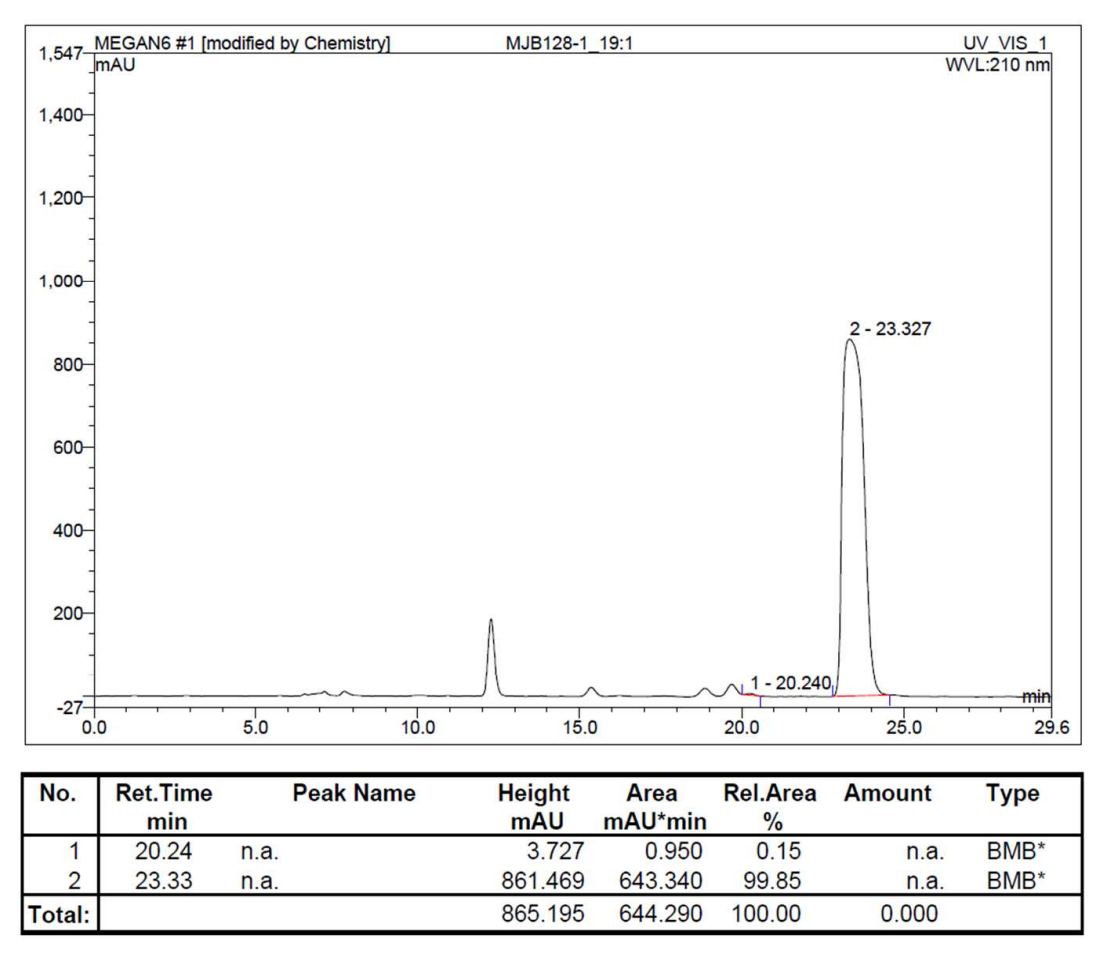


TBDPSO

( \pm -S4

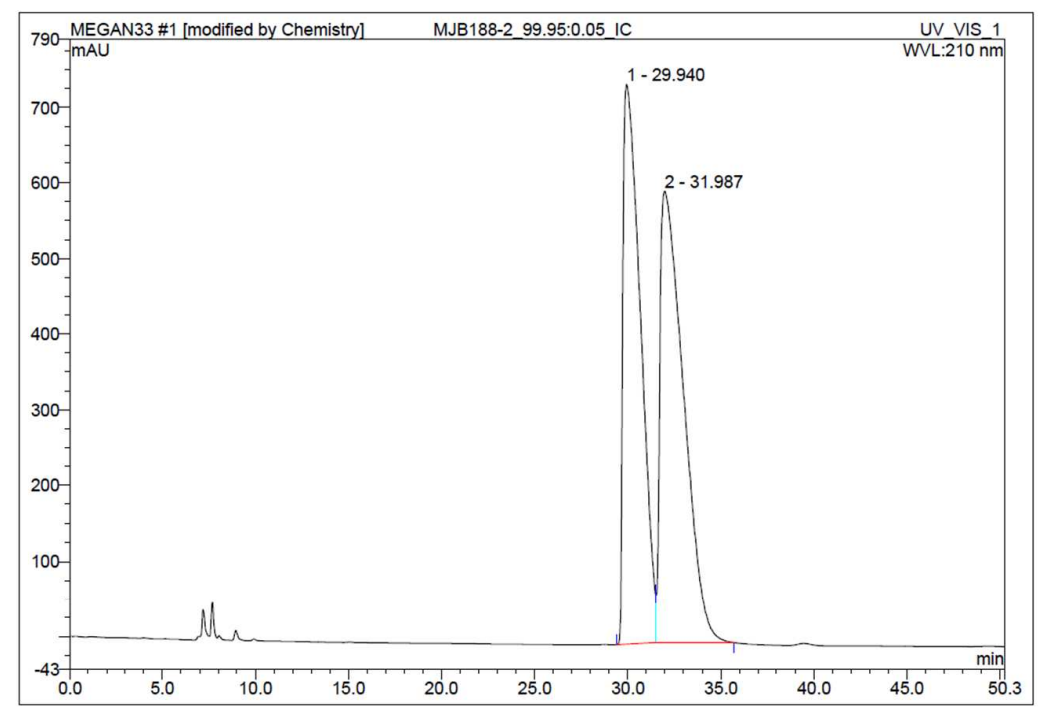

\begin{tabular}{|c|c|c|c|c|c|c|c|}
\hline \begin{tabular}{|l} 
No. \\
\end{tabular} & $\begin{array}{c}\text { Ret.Timte } \\
\text { min }\end{array}$ & Peak Name & $\begin{array}{c}\text { Height } \\
\text { mAU }\end{array}$ & $\begin{array}{c}\text { Area } \\
\text { mAU }\end{array}$ & $\begin{array}{c}\text { Rel.Area } \\
\%\end{array}$ & Amount & Type \\
\hline & $\begin{array}{l}29.94 \\
31.99\end{array}$ & n.a. & $\begin{array}{l}740.676 \\
596585\end{array}$ & $\begin{array}{l}806.810 \\
874853\end{array}$ & $\begin{array}{l}47.98 \\
502\end{array}$ & n.a. & $\mathrm{BM}^{*}$ \\
\hline$\frac{2}{\text { Total: }}$ & 31.99 & n.a. & $\begin{array}{r}596.585 \\
1337.261\end{array}$ & $\begin{array}{r}874.853 \\
1681.663\end{array}$ & $\begin{array}{r}52.02 \\
100.00\end{array}$ & $\begin{array}{r}\text { n.a. } \\
0.000\end{array}$ & $\mathrm{MB}^{\circ}$ \\
\hline
\end{tabular}

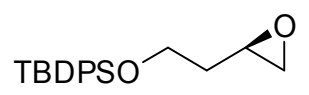

(R)-S4

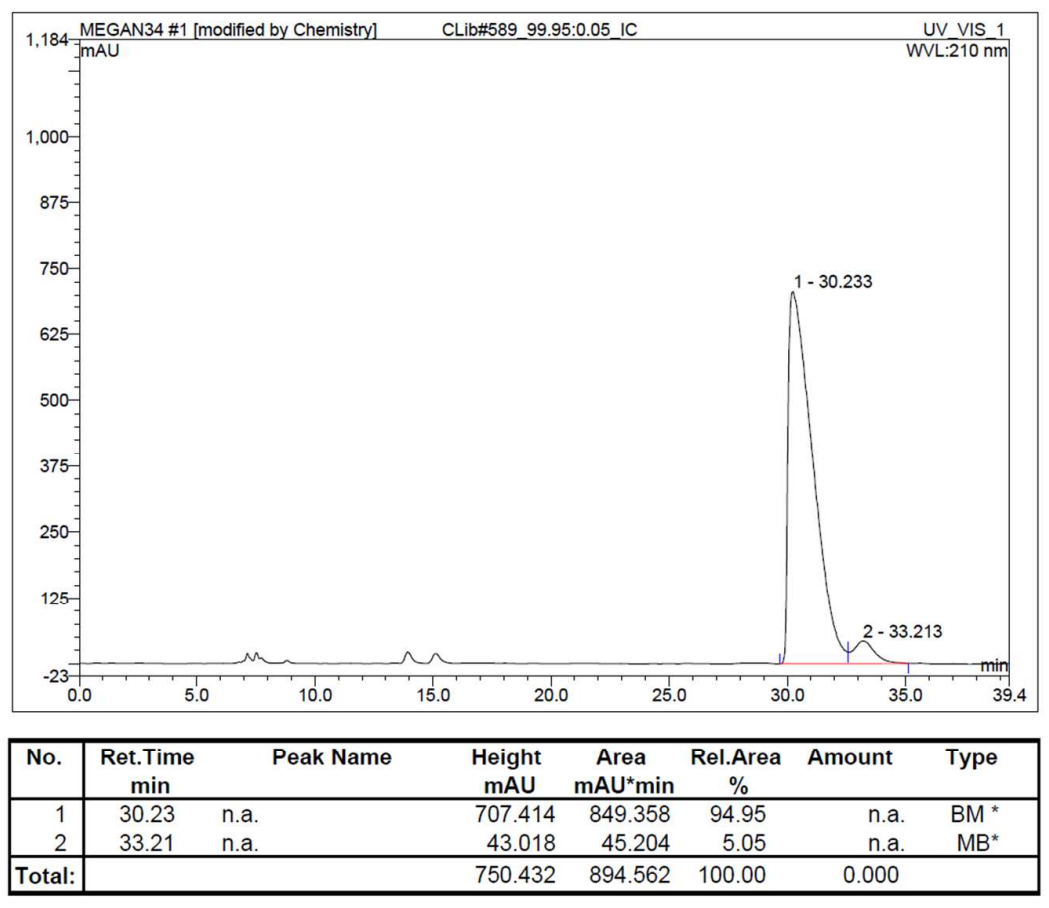




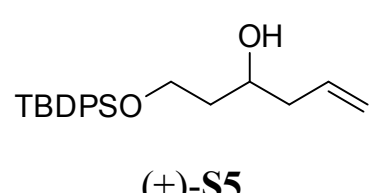

$( \pm)-$ S5



\begin{tabular}{|r|ccccccc|}
\hline No. & $\begin{array}{c}\text { Ret.Time } \\
\text { min }\end{array}$ & Peak Name & $\begin{array}{c}\text { Height } \\
\text { mAU }\end{array}$ & $\begin{array}{c}\text { Area } \\
\mathrm{mAU}^{*} \min \end{array}$ & $\begin{array}{c}\text { Rel.Area } \\
\%\end{array}$ & Amount & Type \\
\hline 1 & 18.25 & n.a. & 374.197 & 156.507 & 48.23 & n.a. & BMB $^{*}$ \\
2 & 21.20 & n.a. & 264.662 & 167.970 & 51.77 & n.a. & BMB $^{*}$ \\
\hline Total: & & & 638.859 & 324.477 & 100.00 & 0.000 & \\
\hline
\end{tabular}

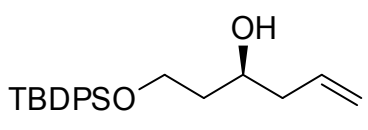

(R)-S5

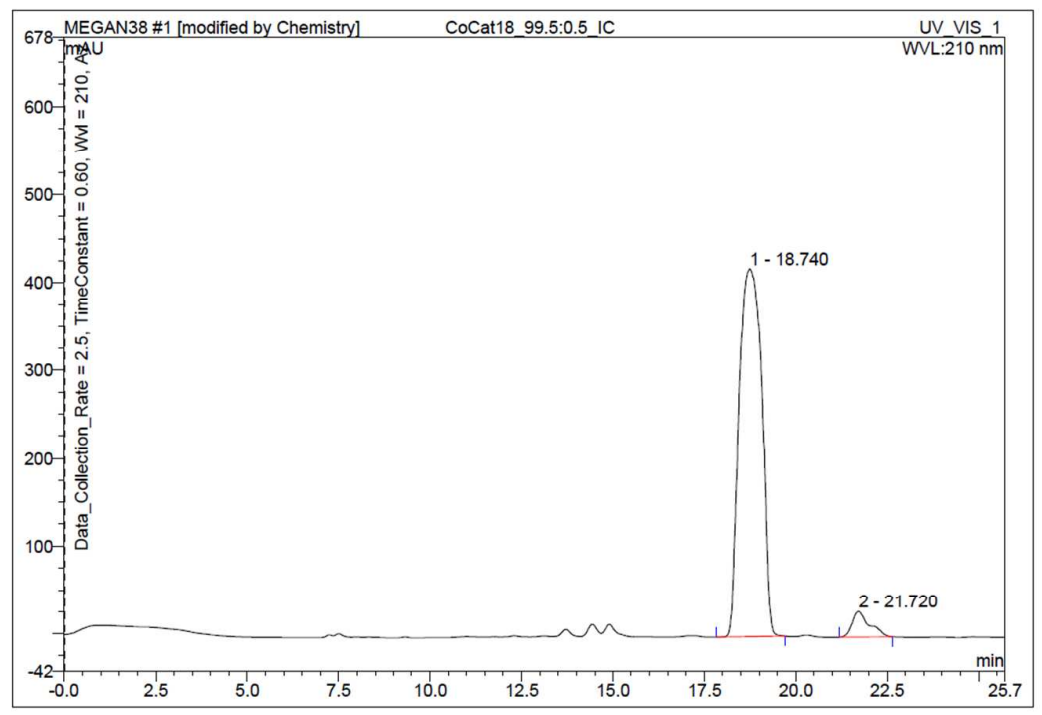

\begin{tabular}{|r|rlrrrrr|}
\hline No. & $\begin{array}{c}\text { Ret.Time } \\
\text { min }\end{array}$ & Peak Name & $\begin{array}{c}\text { Height } \\
\text { mAU }\end{array}$ & $\begin{array}{c}\text { Area } \\
\mathrm{mAU}^{*} \min \end{array}$ & $\begin{array}{c}\text { Rel.Area } \\
\%\end{array}$ & Amount & Type \\
\hline 1 & 18.74 & n.a. & 419.257 & 298.961 & 94.92 & n.a. & BMB $^{*}$ \\
2 & 21.72 & n.a. & 29.045 & 16.003 & 5.08 & n.a. & BMB $^{*}$ \\
\hline Total: & & & 448.303 & 314.963 & 100.00 & 0.000 & \\
\hline
\end{tabular}




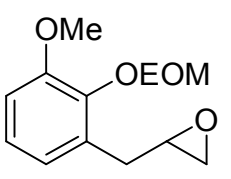

( $)$-S7

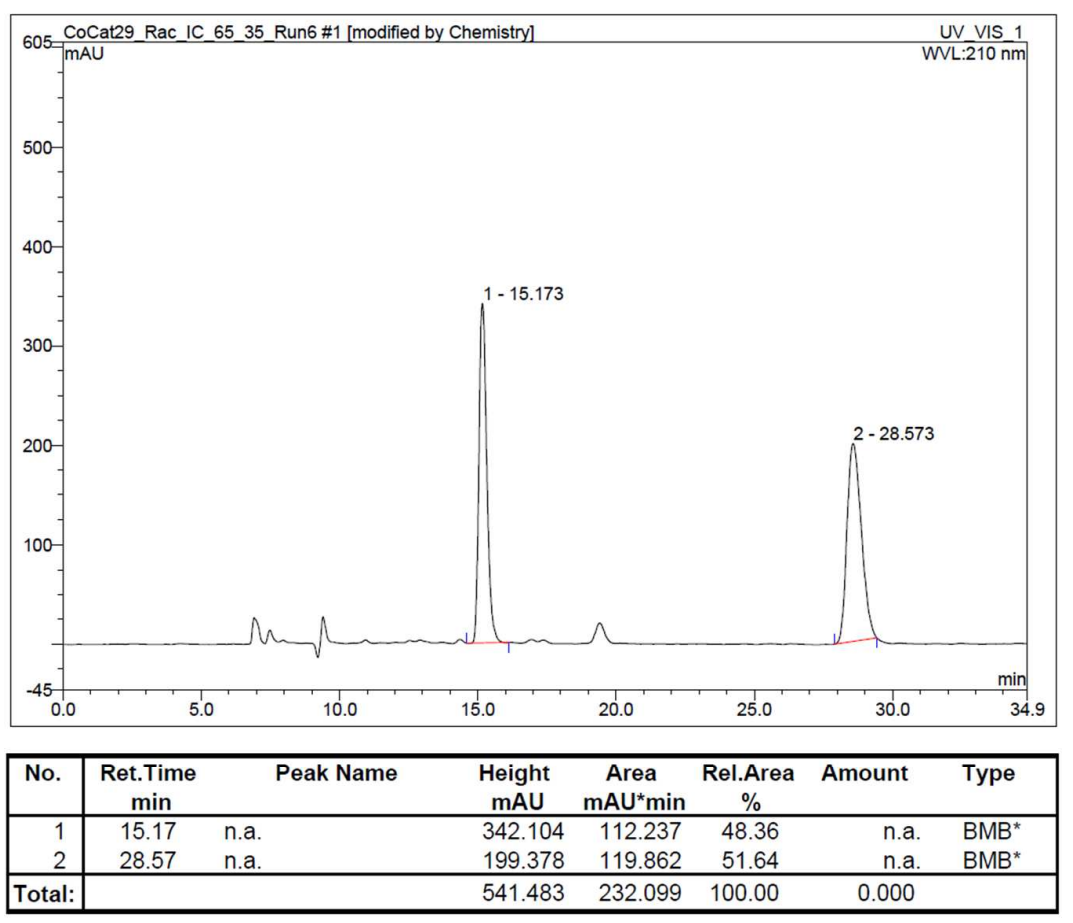



(S)-S7

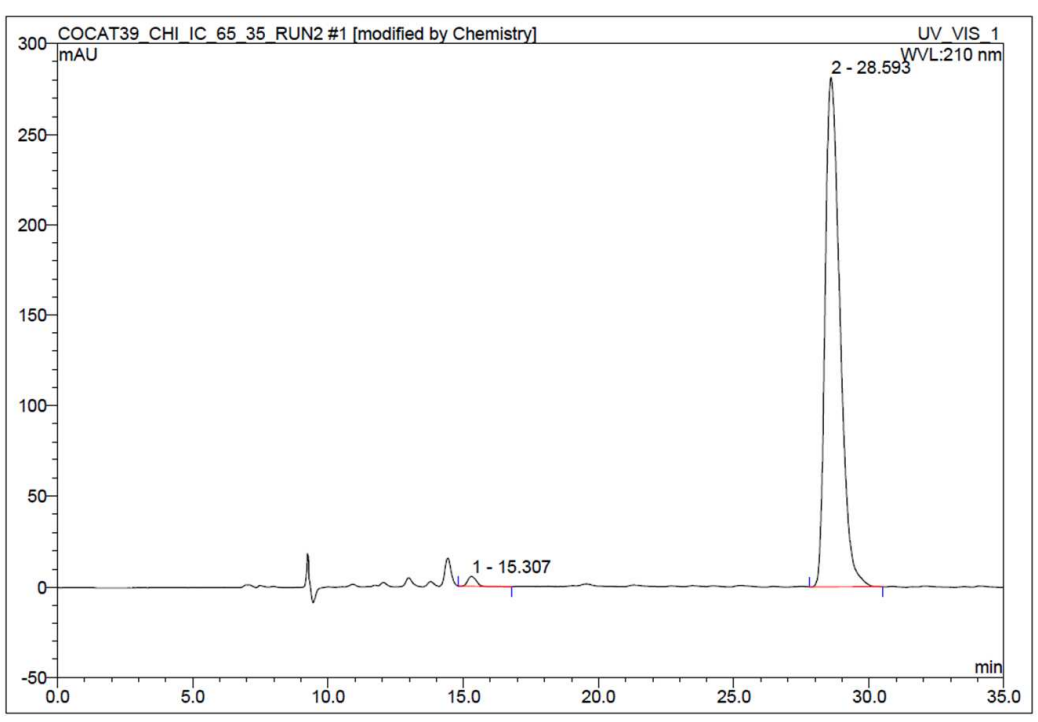

\begin{tabular}{|r|ccrrrrr|}
\hline No. & $\begin{array}{c}\text { Ret.Time } \\
\min \end{array}$ & Peak Name & $\begin{array}{c}\text { Height } \\
\mathrm{mAU}\end{array}$ & $\begin{array}{c}\text { Area } \\
\mathrm{mAU} \text { min }\end{array}$ & $\begin{array}{c}\text { Rel.Area } \\
\%\end{array}$ & Amount & Type \\
\hline 1 & 15.31 & n.a. & 5.360 & 1.858 & 1.03 & n.a. & $\mathrm{BMB}^{*}$ \\
2 & 28.59 & n.a. & 281.169 & 178.381 & 98.97 & n.a. & BMB $^{*}$ \\
\hline Total: & & & 286.528 & 180.239 & 100.00 & 0.000 & \\
\hline
\end{tabular}






( \pm -S8

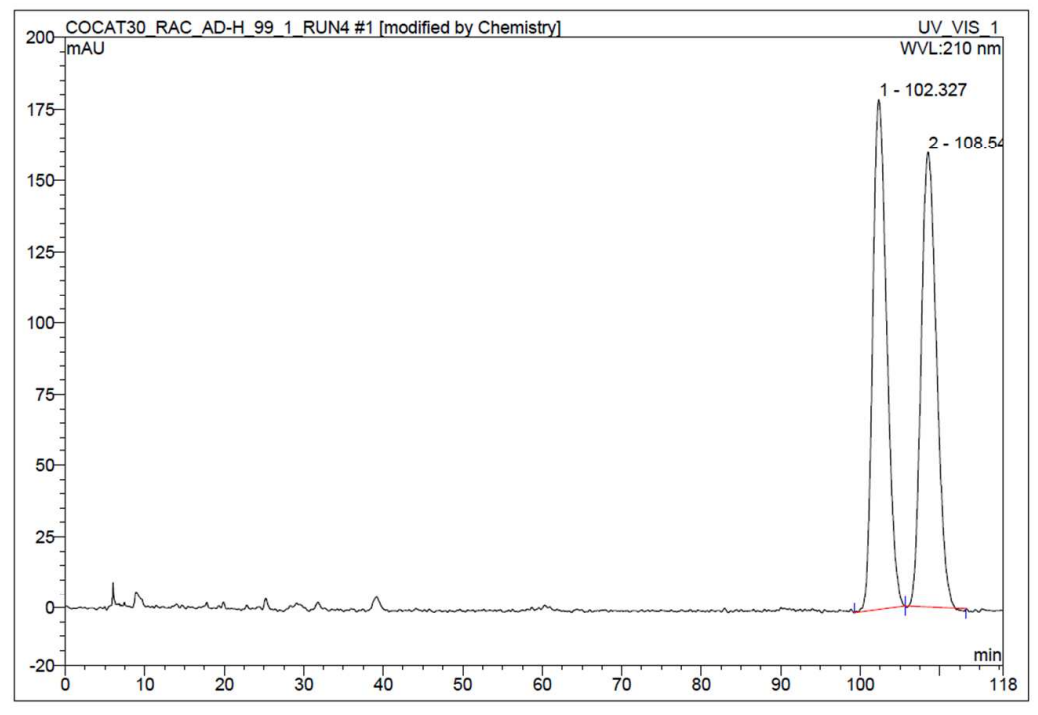

\begin{tabular}{|r|ccccccc|}
\hline No. & $\begin{array}{c}\text { Ret.Time } \\
\text { min }\end{array}$ & Peak Name & $\begin{array}{c}\text { Height } \\
\text { mAU }\end{array}$ & $\begin{array}{c}\text { Area } \\
\text { mAU*min }\end{array}$ & $\begin{array}{c}\text { Rel.Area } \\
\%\end{array}$ & Amount & Type \\
\hline 1 & 102.33 & n.a. & 179.034 & 366.893 & 50.57 & n.a. & BMb $^{*}$ \\
2 & 108.55 & n.a. & 159.665 & 358.618 & 49.43 & n.a. & bMB $^{*}$ \\
\hline Total: & & & 338.699 & 725.511 & 100.00 & 0.000 & \\
\hline
\end{tabular}

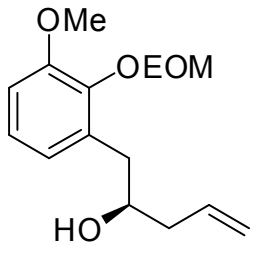

(R)-S8

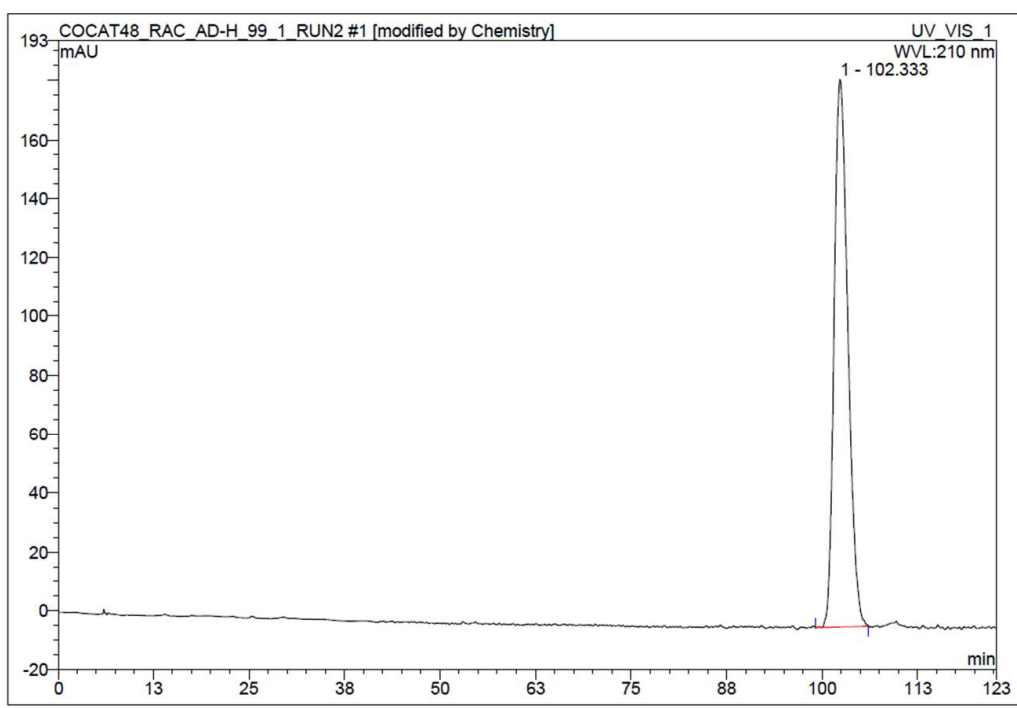

\begin{tabular}{|c|ccccccc|}
\hline No. & $\begin{array}{c}\text { Ret.Time } \\
\min \end{array}$ & Peak Name & $\begin{array}{c}\text { Height } \\
\mathrm{mAU}\end{array}$ & $\begin{array}{c}\text { Area } \\
\mathrm{mAU} \text { min }\end{array}$ & $\begin{array}{c}\text { Rel.Area } \\
\%\end{array}$ & Amount & Type \\
\hline 1 & 102.33 & n.a. & 185.777 & 387.003 & 100.00 & n.a. & BMB $^{*}$ \\
\hline Total: & & & 185.777 & 387.003 & 100.00 & 0.000 & \\
\hline
\end{tabular}

